九州大学学術情報リポジトリ

Kyushu University Institutional Repository

Large time behavior of solutions to the compressible Navier-Stokes equations in an infinite layer under slip boundary condition

Aihaiti, Abulizi

Graduate School of Mathematics, Kyushu University

Enomoto, Shota

Graduate School of Mathematics, Kyushu University

Kage i, Yoshiyuki

Faculty of Mathematics, Kyushu University

http://hdl. handle. net/2324/1661897

出版情報 : MI Preprint Series. 2016-8，2016-05-21. 九州大学大学院数理学研究院 バージョン：

権利関係 : 


\section{Preprint Series}

Mathematics for Industry Kyushu University

\section{Large time behavior of solutions to the compressible Navier-Stokes equations in an infinite layer under slip boundary condition}

\section{Abulizi Aihaiti, Shota Enomoto \& Yoshiyuki Kagei \\ MI 2016-8}

( Received May 21, 2016 )

Institute of Mathematics for Industry

Graduate School of Mathematics

Kyushu University

Fukuoka, JAPAN 


\title{
Large time behavior of solutions to the compressible Navier-Stokes equations in an infinite layer under slip boundary condition
}

\author{
Abulizi Aihaiti $^{1}$, Shota Enomoto ${ }^{1}$, Yoshiyuki Kagei ${ }^{2}$ \\ ${ }^{1}$ Graduate School of Mathematics, \\ Kyushu University, \\ Nishi-ku, Motooka 744, \\ Fukuoka 819-0395, Japan \\ 2 Faculty of Mathematics, \\ Kyushu University, \\ Nishi-ku, Motooka 744, \\ Fukuoka 819-0395, Japan
}

\begin{abstract}
This paper is concerned with large time behavior of solutions to the compressible Navier-Stokes equations in an infinite layer of $\mathbb{R}^{2}$ under slip boundary condition. It is shown that if the initial data is sufficiently small, the global solution uniquely exists and the large time behavior of the solution is described by a superposition of onedimensional diffusion waves.
\end{abstract}

Keywords: Compressible Navier-Stokes equation, infinite layer, slip boundary condition, asymptotic behavior, diffusion waves.

\section{Introduction}

This paper studies large time behavior of solutions of the compressible NavierStokes equation

$$
\begin{gathered}
\partial_{t} \rho+\operatorname{div}(\rho v)=0 \\
\rho\left(\partial_{t} v+v \cdot \nabla v\right)-\mu \Delta v-\left(\mu+\mu^{\prime}\right) \nabla \operatorname{div} v+\nabla P(\rho)=0
\end{gathered}
$$

in an infinite layer $\Omega$ of $\mathbb{R}^{2}$ :

$$
\Omega=\left\{x=\left(x_{1}, x_{2}\right) \in \mathbb{R}^{2} ; x_{1} \in \mathbb{R}, 0<x_{2}<1\right\}
$$


under the slip boundary condition

$$
\left.\partial_{x_{2}} v^{1}\right|_{x_{2}=0,1}=0,\left.\quad v^{2}\right|_{x_{2}=0,1}=0 .
$$

Here $\rho=\rho(x, t)>0$ and $v={ }^{\top}\left(v^{1}(x, t), v^{2}(x, t)\right)$ denote the unknown density and velocity, respectively, at time $t \geq 0$ and position $x \in \Omega ; P=P(\rho)$ is the pressure that is assumed to be a smooth function of $\rho$ satisfying

$$
P^{\prime}\left(\rho_{*}\right)>0
$$

for a given constant $\rho_{*}>0 ; \mu$ and $\mu^{\prime}$ are viscosity coefficients that are assumed to be constants and satisfy

$$
\mu>0, \quad \mu+\mu^{\prime} \geq 0
$$

div, $\nabla$ and $\Delta$ denote the usual divergence, gradient and Laplacian with respect to $x$. Here and in what follows ${ }^{\top}$. means the transposition.

We impose the initial condition

$$
\left.\rho\right|_{t=0}=\rho_{0},\left.\quad v\right|_{t=0}=v_{0} .
$$

Here $\rho_{0}=\rho_{0}(x)$ and $v_{0}=v_{0}(x)$ satisfy $\rho_{0}(x) \rightarrow \rho_{*}$ and $v_{0}(x) \rightarrow 0$ as $|x| \rightarrow \infty$.

The aim of this paper is to investigate large time behavior of solutions to (1.1)-(1.4) around the motionless state $\rho=\rho_{*}, v=0$. We rewrite (1.1)-(1.2) into the following equations for the perturbation

$$
\begin{gathered}
\partial_{t} \phi+\gamma \operatorname{div} w=f^{0}(\phi, w), \\
\partial_{t} w-\nu \Delta w-\tilde{\nu} \nabla \operatorname{div} w+\gamma \nabla \phi=\tilde{f}(\phi, w) .
\end{gathered}
$$

Here $u={ }^{\top}(\phi, w)$ with $\phi=\frac{1}{\rho_{*}}\left(\rho-\rho_{*}\right)$ and $w=\frac{1}{\gamma} v$ denotes the perturbation from $u_{s}={ }^{\top}\left(\rho_{*}, 0\right) ; \nu, \tilde{\nu}$ and $\gamma$ are parameters given by

$$
\nu=\frac{\mu}{\rho_{*}}, \quad \tilde{\nu}=\frac{\mu+\mu^{\prime}}{\rho_{*}}, \quad \gamma=\sqrt{P^{\prime}\left(\rho_{*}\right)} ;
$$

and $f(\phi, w)={ }^{\top}\left(f^{0}(\phi, w), \tilde{f}(\phi, w)\right)$ denote the nonlinear terms:

$$
\begin{aligned}
f^{0}(\phi, w)= & -\gamma \operatorname{div}(\phi w), \\
\tilde{f}(\phi, w)= & -\gamma w \cdot \nabla w-\frac{\phi}{1+\phi}\{\nu \Delta w+\tilde{\nu} \nabla \operatorname{div} w\}+\frac{\gamma \phi}{1+\phi} \nabla \phi \\
& -\frac{\rho_{*}}{\gamma(1+\phi)} \nabla\left(P^{(2)}(\phi) \phi^{2}\right),
\end{aligned}
$$


where

$$
P^{(2)}(\phi)=\int_{0}^{1}(1-\theta) P^{\prime \prime}\left(\rho_{*}(1+\theta \phi)\right) \mathrm{d} \theta .
$$

The boundary condition (1.3) and initial condition (1.4) are transformed into

$$
\left.\partial_{x_{2}} w^{1}\right|_{x_{2}=0,1}=0,\left.\quad w^{2}\right|_{x_{2}=0,1}=0
$$

and

$$
\left.u\right|_{t=0}=u_{0}={ }^{\top}\left(\phi_{0}, w_{0}\right) .
$$

Here $u_{0}$ satisfies $u_{0}(x) \rightarrow 0$ as $|x| \rightarrow \infty$.

The large time behavior of solutions of the compressible Navier-Stokes equation (1.1)-(1.2) on the layer $\Omega$ was studied in $[1,2,3,4]$ under the nonslip boundary condition $\left.v\right|_{x_{2}=0,1}=0$. It was shown in [3] that the large time behavior of perturbations of the motionless state is described by a one-dimensional linear heat equation. In [4] stability of parallel flow was considered and it was proved that the large time behavior of perturbations of parallel flow is described by a one-dimensional viscous Burgers equation when the Reynolds and Mach numbers are sufficiently small. In the case of time-periodic parallel flow, the large time behavior of perturbations is also described by a one-dimensional diffusion equation $([1,2])$. In all cases of $[1,2,3,4]$, the asymptotic leading parts under the non-slip boundary condition exhibit purely diffusive phenomena. In this paper we show that the solution of (1.1)-(1.2) under the slip boundary condition (1.3) with (1.4) behaves like a superposition of one-dimensional diffusion waves as $t \rightarrow \infty$ as in the case of one-dimensional compressible Navier-Stokes equation [7, 10]. More precisely, consider problem (1.5)-(1.8) for $u$. We prove that, under appropriate conditions for $u_{0}$, the solution $u(t)$ satisfies

$$
\left\|\partial_{x}^{k}\left(u-\chi_{+} \boldsymbol{a}_{+}-\chi_{-} \boldsymbol{a}_{-}\right)(t)\right\|_{L^{2}} \leq C(1+t)^{-\frac{1}{2}-\frac{k}{2}}, \quad k=0,1,
$$

where $\boldsymbol{a}_{ \pm}={ }^{\top}(1, \pm 1,0)$ and $\chi_{ \pm}=\chi_{ \pm}\left(x_{1}, t\right)$ are the diffusion waves given by

$$
\chi_{ \pm}\left(x_{1}, t\right)=z_{ \pm}\left(x_{1} \pm \gamma t, t\right) .
$$

Here $z_{ \pm}=z_{ \pm}\left(x_{1}, t\right)$ are the self-similar solutions of the viscous Burgers equations

$$
\partial_{t} z_{ \pm}-\frac{\nu+\tilde{\nu}}{2} \partial_{x_{1}}^{2} z_{ \pm} \mp c \partial_{x_{1}}\left(z_{ \pm}^{2}\right)=0
$$

satisfying

$$
\int_{\mathbb{R}} z_{ \pm}\left(x_{1}, t\right) \mathrm{d} x_{1}=\frac{1}{2} \int_{\Omega}\left(\phi_{0}(x) \pm\left(1+\phi_{0}(x)\right) w_{0}^{1}(x)\right) \mathrm{d} x
$$


for some constant $c \in \mathbb{R}$. In contrast to the case of the non-slip boundary condition, we see that a hyperbolic aspect of (1.1)-(1.2) appears in the asymptotic leading part of the solution under the slip boundary condition.

To prove (1.9), we first establish the decay estimates for $u(t)$. We decompose the solution of (1.5)-(1.8) into its low and high frequency parts. The spectrum of the low-frequency part of the linearized semigroup is different from the one in the case of the non-slip boundary condition; it is the same as that in the case of the one-dimensional compressible Navier-Stokes equation. Therefore, the low-frequency part decays like one-dimensional heat kernel, namely, $k$ th order derivative decays in the order $O\left(t^{-\frac{1}{4}-\frac{k}{2}}\right)$ in the $L^{2}$ norm. For the high-frequency part (remainder part), we apply the MatsumuraNishida energy method ([9]) to see that the high-frequency part decays in the order $O\left(t^{-\frac{5}{4}}\right)$ in the $H^{2}$ norm. Based on the spectral properties of the low-frequency part of the linearized semigroup and the decay estimate for the high-frequency part, we deduce the asymptotic behavior (1.9) by applying the argument of Kawashima [7].

The paper is organized as follows. In section 2 we state the main results of this paper. In section 3 we study the spectral properties of the linearized operator, and in section 4 we rewrite (1.5)-(1.8) into a problem for a system of equations for the low and high frequency parts. Section 5 is devoted to estimating the low-frequency part, while the high-frequency part is estimated in section 6 . In section 7 we give the estimates for the nonlinear terms. In section 8 we study the asymptotic behavior of the solution of (1.5)-(1.8).

\section{Main Results}

In this section we state the main results of this paper. We first introduce notation and function spaces which will be used throughout this paper.

For $1 \leq p \leq \infty$ we denote by $L^{p}(X)$ the usual Lebesgue space on a domain $X$ and its norm is denoted by $\|\cdot\|_{L^{p}(X)}$. Let $m$ be a nonnegative integer. The symbol $H^{m}(X)$ denotes the $m$-th order $L^{2}$-Sobolev space on $X$ with norm $\|\cdot\|_{H^{m}(X)}$. In particular, we write $\|\cdot\|_{L^{2}(X)}$ for $H^{0}(X)$.

We simply denote by $L^{p}(X)$ (resp., $H^{m}(X)$ ) the set of all vector fields $w={ }^{\top}\left(w^{1}, w^{2}\right)$ on $X$ with $w^{j} \in L^{p}(X)\left(\operatorname{resp}, H^{m}(X)\right), j=1,2$, and its norm is also denoted by $\|\cdot\|_{L^{p}(X)}$ (resp., $\left.\|\cdot\|_{H^{m}(X)}\right)$. For $u={ }^{\top}(\phi, w)$ with $\phi \in H^{k}(X)$ and $w={ }^{\top}\left(w^{1}, w^{2}\right) \in H^{m}(X)$, we define $\|u\|_{H^{k}(X) \times H^{m}(X)}$ by $\|u\|_{H^{k}(X) \times H^{m}(X)}=\|\phi\|_{H^{k}(X)}+\|w\|_{H^{k}(X)}$. When $k=m$, we simply write $\|u\|_{H^{k}(X) \times H^{k}(X)}=\|u\|_{H^{k}(X)}$.

Partial derivatives of a function $u$ in $x, x_{k}(k=1,2)$ and $t$ are denoted by $\partial_{x} u, \partial_{x_{k}} u$ and $\partial_{t} u$. We also write the higher order partial derivatives of $u$ 
in $x$ as $\partial_{x}^{l} u=\left(\partial_{x}^{\alpha} u ;|\alpha|=l\right)$.

In the case where $X=\Omega$ we abbreviate $L^{p}(\Omega)$ (resp., $H^{m}(\Omega)$ ) as $L^{p}$ (resp., $H^{m}$ ). In particular, the norm $\|\cdot\|_{L^{p}(\Omega)}=\|\cdot\|_{L^{p}}$ is denoted by $\|\cdot\|_{p}$. We denote the inner product of $L^{2}(\Omega)$ by

$$
(f, g)=\int_{\Omega} f(x) g(x) \mathrm{d} x, \quad f, g \in L^{2}(\Omega) .
$$

The average of a function $f$ in $x_{2}$ on $(0,1)$ is denoted by $\langle f\rangle$ :

$$
\langle f\rangle=\int_{0}^{1} f\left(x_{2}\right) \mathrm{d} x_{2} .
$$

We set

$$
H_{*}^{2}=\left\{w={ }^{\top}\left(w^{1}, w^{2}\right) \in H^{2}(\Omega) ;\left.\partial_{x_{2}} w^{1}\right|_{x_{2}=0,1}=0,\left.w^{2}\right|_{x_{2}=0,1}=0\right\} .
$$

For $\alpha \in \mathbb{R}$, we denote by $L_{\alpha}^{1}=L_{\alpha}^{1}(\Omega)$ the weighted $L^{1}$ space with weight $\left(1+\left|x_{1}\right|\right)^{\alpha}$, and its norm is denoted by

$$
\|f\|_{L_{\alpha}^{1}}=\int_{\Omega}\left(1+\left|x_{1}\right|\right)^{\alpha}|f(x)| \mathrm{d} x .
$$

We denote the Fourier transform of $f=f\left(x_{1}\right)\left(x_{1} \in \mathbb{R}\right)$ by $\hat{f}$ or $\mathcal{F}[f]$ :

$$
\hat{f}(\xi)=\mathcal{F}[f](\xi)=\int_{\mathbb{R}} f\left(x_{1}\right) e^{-i \xi x_{1}} \mathrm{~d} x_{1}, \quad \xi \in \mathbb{R} .
$$

The inverse Fourier transform is denoted by $\mathcal{F}^{-1}$ :

$$
\mathcal{F}^{-1}[f]\left(x_{1}\right)=(2 \pi)^{-1} \int_{\mathbb{R}} f(\xi) e^{i \xi x_{1}} \mathrm{~d} \xi, \quad x_{1} \in \mathbb{R} .
$$

For operators $A, B$, we denote the commutator of $A$ and $B$ by $[A, B]$ :

$$
[A, B] f=A(B f)-B(A f) .
$$

We now state the main results of this paper. We have the following decay estimate of the $L^{2}$ norm of the solution $u$.

Theorem 2.1 There exists a positive number $\varepsilon_{0}$ such that if $u_{0}={ }^{\top}\left(\phi_{0}, w_{0}\right) \in$ $\left(H^{2} \times H_{*}^{2}\right) \cap L^{1}$ with $w_{0}={ }^{\top}\left(w_{0}^{1}, w_{0}^{2}\right)$ satisfies $\left\|u_{0}\right\|_{H^{2} \cap L^{1}} \leq \varepsilon_{0}$, then problem (1.5)-(1.8) has a unique global solution

$$
u(t)={ }^{\top}(\phi(t), w(t)) \in C\left([0, \infty) ; H^{2} \times H_{*}^{2}\right)
$$

and $u(t)$ satisfies

$$
\left\|\partial_{x}^{k} u(t)\right\|_{2} \leq C(1+t)^{-\frac{1}{4}-\frac{k}{2}}\left\|u_{0}\right\|_{H^{2} \cap L^{1}}
$$

for $t \geq 0, k=0,1,2$. 
We next consider the asymptotic behavior of solutions.

Theorem 2.2 In addition to the assumption of Theorem 2.1, if $\phi_{0}, w_{0}^{1} \in$ $L_{1 / 2}^{1}$, then

$$
\left\|\partial_{x}^{k}\left(u-\chi_{+} \boldsymbol{a}_{+}-\chi_{-} \boldsymbol{a}_{-}\right)(t)\right\|_{2} \leq C(1+t)^{-\frac{1}{2}-\frac{k}{2}}, \quad k=0,1 .
$$

Here $\boldsymbol{a}_{ \pm}={ }^{\top}(1, \pm 1,0)$ and $\chi_{ \pm}=\chi_{ \pm}\left(x_{1}, t\right)$ are the diffusion waves given in (1.10)-(1.12).

The proof of Theorem 2.1 will be given in sections $3-7$, and Theorem 2.2 will be proved in section 8 .

\section{Spectral Properties of Linearized Operator}

We consider the linearized problem

$$
\partial_{t} u+L u=F,\left.\quad u\right|_{t=0}=u_{0},
$$

where $u={ }^{\top}(\phi, w) ; F={ }^{\top}\left(f^{0}, \tilde{f}\right)$ with $\tilde{f}={ }^{\top}\left(f^{1}, f^{2}\right)$ is a given function, and $L$ is an operator of the form

$$
L=\left(\begin{array}{cc}
0 & \gamma \operatorname{div} \\
\gamma \nabla & -\nu \Delta-\tilde{\nu} \nabla \operatorname{div}
\end{array}\right)
$$

in $H^{1} \times L^{2}$ with domain $D(L)=H^{1} \times H_{*}^{2}$.

To investigate (3.1), we consider the Fourier transform of (3.1) in $x_{1} \in \mathbb{R}$ :

$$
\begin{gathered}
\partial_{t} \hat{\phi}+i \gamma \xi \hat{w}^{1}+\gamma \partial_{x_{2}} \hat{w}^{2}=\hat{f}^{0} \\
\partial_{t} \hat{w}^{1}+(\nu+\tilde{\nu}) \xi^{2} \hat{w}^{1}-\nu \partial_{x_{2}}^{2} \hat{w}^{1}-i \tilde{\nu} \xi \partial_{x_{2}} \hat{w}^{2}+i \gamma \xi \hat{\phi}=\hat{f}^{1} \\
\partial_{t} \hat{w}^{2}+\nu \xi^{2} \hat{w}^{2}-(\nu+\tilde{\nu}) \partial_{x_{2}}^{2} \hat{w}^{2}-i \tilde{\nu} \xi \partial_{x_{2}} \hat{w}^{1}+\gamma \partial_{x_{2}} \hat{\phi}=\hat{f}^{2} \\
\left.\partial_{x_{2}} \hat{w}^{1}\right|_{x_{2}=0,1}=\left.\hat{w}^{2}\right|_{x_{2}=0,1}=0 \\
\left.\hat{u}\right|_{t=0}=\hat{u}_{0}={ }^{\top}\left(\hat{\phi}_{0}, \hat{w}_{0}\right) .
\end{gathered}
$$

We thus arrive at the following problem

$$
\partial_{t} \hat{u}+\hat{L}_{\xi} \hat{u}=\hat{F},\left.\quad \hat{u}\right|_{t=0}=\hat{u}_{0},
$$

with a parameter $\xi \in \mathbb{R}$. Here $\hat{u}=\hat{u}\left(\xi, x_{2}, t\right) ; \hat{L}_{\xi}$ is the operator

$$
\hat{L}_{\xi}=\left(\begin{array}{ccc}
0 & i \gamma \xi & \gamma \partial_{x_{2}} \\
i \gamma \xi & (\nu+\tilde{\nu}) \xi^{2}-\nu \partial_{x_{2}}^{2} & -i \tilde{\nu} \xi \partial_{x_{2}} \\
\gamma \partial_{x_{2}} & -i \tilde{\nu} \xi \partial_{x_{2}} & \nu \xi^{2}-(\nu+\tilde{\nu}) \partial_{x_{2}}^{2}
\end{array}\right)
$$

with domain $D\left(\hat{L}_{\xi}\right)=H^{1}(0,1) \times H_{*}^{2}(0,1)$, where $H_{*}^{2}(0,1)=\left\{w={ }^{\top}\left(w^{1}, w^{2}\right) \in\right.$ $\left.H^{2}(0,1) ;\left.\partial_{x_{2}} w^{1}\right|_{x_{2}=0,1}=\left.w^{2}\right|_{x_{2}=0,1}=0\right\}$. For $-\hat{L}_{0}$ we have the following result. 
Lemma 3.1 (i) $\lambda=0$ is a semisimple eigenvalue of $-\hat{L}_{0}$.

(ii) The eigenprojection $\Pi$ for $\lambda=0$ of $-\hat{L}_{0}$ is given by

$$
\Pi u=\left(\begin{array}{c}
\langle\phi\rangle \\
\left\langle w^{1}\right\rangle \\
0
\end{array}\right)
$$

for $u={ }^{\top}(\phi, w)$ with $w={ }^{\top}\left(w^{1}, w^{2}\right)$.

The proof of Lemma 3.1 is straightforward and we omit it.

We next expand $\hat{u}$ and $\hat{F}$ into the Fourier series:

$$
\begin{aligned}
& \hat{\phi}=\sum_{k=0}^{\infty} \hat{\phi}_{k} \cos k \pi x_{2}, \hat{w}^{1}=\sum_{k=0}^{\infty} \hat{w}_{k}^{1} \cos k \pi x_{2}, \hat{w}^{2}=\sum_{k=1}^{\infty} \hat{w}_{k}^{2} \sin k \pi x_{2}, \\
& \hat{f}^{0}=\sum_{k=0}^{\infty} \hat{f}_{k}^{0} \cos k \pi x_{2}, \hat{f}^{1}=\sum_{k=0}^{\infty} \hat{f}_{k}^{1} \cos k \pi x_{2}, \hat{f}^{2}=\sum_{k=1}^{\infty} \hat{f}_{k}^{2} \sin k \pi x_{2} .
\end{aligned}
$$

It then follows that

$$
\begin{gathered}
\partial_{t} \hat{\phi}_{k}+i \gamma \xi \hat{w}_{k}^{1}+\gamma \hat{w}_{k}^{2} k \pi=\hat{f}_{k}^{0}, \\
\partial_{t} \hat{w}_{k}^{1}+\nu\left(\xi^{2}+k^{2} \pi^{2}\right) \hat{w}_{k}^{1}+\tilde{\nu} \xi^{2} \hat{w}_{k}^{1}-i \tilde{\nu} k \pi \xi \hat{w}_{k}^{2}+i \gamma \xi \hat{\phi}_{k}=\hat{f}_{k}^{1}, \\
\partial_{t} \hat{w}_{k}^{2}+\nu\left(\xi^{2}+k^{2} \pi^{2}\right) \hat{w}_{k}^{2}+i \tilde{\nu} k \pi \xi \hat{w}_{k}^{1}+\tilde{\nu} k^{2} \pi^{2} \hat{w}_{k}^{2}-\gamma k \pi \hat{\phi}_{k}=\hat{f}_{k}^{2} .
\end{gathered}
$$

We rewrite it in the form

$$
\partial_{t} \hat{u}_{k}+\hat{L}_{\xi, k} \hat{u}_{k}=\hat{F}_{k}
$$

where $\hat{u}_{k}={ }^{\top}\left(\hat{\phi}_{k}, \hat{w}_{k}^{1}, \hat{w}_{k}^{2}\right), \hat{F}_{k}={ }^{\top}\left(\hat{f}_{k}^{0}, \hat{f}_{k}^{1}, \hat{f}_{k}^{2}\right)$ and

$$
\hat{L}_{\xi, k}=\left(\begin{array}{ccc}
0 & i \gamma \xi & \gamma k \pi \\
i \gamma \xi & \nu\left(\xi^{2}+k^{2} \pi^{2}\right)+\tilde{\nu} \xi^{2} & -i \tilde{\nu} k \pi \xi \\
-\gamma k \pi & i \tilde{\nu} k \pi \xi & \nu\left(\xi^{2}+k^{2} \pi^{2}\right)+\tilde{\nu} k^{2} \pi^{2}
\end{array}\right) .
$$

As for the the spectrum of $-\hat{L}_{\xi, k}$, we have the following lemma.

Lemma 3.2 (i) The eigenvalues $-\hat{L}_{\xi, k}$ are given by

$$
\begin{aligned}
\lambda_{0, k}(\xi) & =-\nu\left(\xi^{2}+k^{2} \pi^{2}\right), \\
\lambda_{ \pm, k}(\xi)= & -\frac{1}{2}(\nu+\tilde{\nu})\left(\xi^{2}+k^{2} \pi^{2}\right) \\
& \pm \frac{1}{2} \sqrt{(\nu+\tilde{\nu})^{2}\left(\xi^{2}+k^{2} \pi^{2}\right)^{2}-4 \gamma^{2}\left(\xi^{2}+k^{2} \pi^{2}\right)}
\end{aligned}
$$


(ii) The eigenprojections for $\lambda_{0, k}$ and $\lambda_{ \pm, k}$ are given by the following $P_{0, k}$ and $P_{ \pm, k}$, respectively:

$$
\begin{aligned}
P_{0, k} & =\left(\begin{array}{ccc}
0 & 0 & 0 \\
0 & 1-\frac{\xi^{2}}{\xi^{2}+k^{2} \pi^{2}} & \frac{i k \pi \xi}{\xi^{2}+k^{2} \pi^{2}} \\
0 & -\frac{i k \pi \xi}{\xi^{2}+k^{2} \pi^{2}} & 1-\frac{k^{2} \pi^{2}}{\xi^{2}+k^{2} \pi^{2}}
\end{array}\right), \\
P_{+, k} & =\frac{1}{\lambda_{+, k}-\lambda_{-, k}}\left(\begin{array}{ccc}
-\lambda_{-, k} & i \gamma \xi & \gamma k \pi \\
i \gamma \xi & \frac{\xi^{2} \lambda_{+, k}}{\xi^{2}+k^{2} \pi^{2}} & -\frac{i k \pi \xi \lambda_{+, k}}{\xi^{2}+k^{2} \pi^{2}} \\
-\gamma k \pi & \frac{i k \pi \xi \lambda_{, k}}{\xi^{2}+k^{2} \pi^{2}} & \frac{k^{2} \pi^{2} \lambda_{+, k}}{\xi^{2}+k^{2} \pi^{2}}
\end{array}\right), \\
P_{-, k} & =\frac{1}{\lambda_{+, k}-\lambda_{-, k}}\left(\begin{array}{ccc}
\lambda_{+, k} & -i \gamma \xi & -\gamma k \pi \\
-i \gamma \xi & -\frac{\xi^{2} \lambda_{-, k}}{\xi^{2}+k^{2} \pi^{2}} & \frac{i k \pi \xi \lambda_{-, k}}{\xi^{2}+k^{2} \pi^{2}} \\
\gamma k \pi & -\frac{i k \pi \xi \lambda_{-, k}}{\xi^{2}+k^{2} \pi^{2}} & -\frac{k^{2} \pi^{2} \lambda_{-, k}}{\xi^{2}+k^{2} \pi^{2}}
\end{array}\right) .
\end{aligned}
$$

Lemma 3.2 can be proved by elementary computations.

\section{Decay estimate: Proof of Theorem 2.1}

We consider the nonlinear problem

$$
\left\{\begin{array}{l}
\partial_{t} u+L u=F(u) \\
\left.u\right|_{t=0}=u_{0}
\end{array}\right.
$$

Here $u={ }^{\top}(\phi, w)$ and $F(u)=^{\top}\left(f^{0}(\phi, w), \tilde{f}(\phi, w)\right)$.

One can prove the local solvability for (4.1) as in [5].

Proposition 4.1 Assume that $u_{0}={ }^{\top}\left(\phi_{0}, w_{0}\right) \in H^{2} \times H_{*}^{2}$ and $\left\|\phi_{0}\right\|_{\infty} \leq$ $\frac{1}{2}$. Then there exists $T_{0}>0$ depending on $\left\|u_{0}\right\|_{H^{2}}$ such that problem (4.1) has a unique solution $u={ }^{\top}(\phi, w)$ on $\left[0, T_{0}\right]$ satisfying $u \in C\left(\left[0, T_{0}\right] ; H^{2} \times\right.$ $\left.H_{*}^{2}\right) \cap C^{1}\left(\left[0, T_{0}\right] ; L^{2}\right)$ with $w \in L^{2}\left(0, T_{0} ; H^{3}\right)$ and $\left\|\phi_{0}(t)\right\|_{\infty} \leq \frac{3}{4}$ for $t \in\left[0, T_{0}\right]$. Furthermore, the inequality

$$
\sup _{t \in\left[0, T_{0}\right]}\left\{\|u(t)\|_{H^{2}}+\left\|\partial_{t} u(t)\right\|_{2}\right\}+\int_{0}^{T_{0}}\|w\|_{H^{3}}^{2} \mathrm{~d} t \leq C_{0}\left\{1+\left\|u_{0}\right\|_{H^{2}}^{2}\right\}^{a}\left\|u_{0}\right\|_{H^{2}}^{2}
$$

holds with some constants $C_{0}>0$ and $a>0$.

The global existence of $u(t)$ follows in a standard manner from Proposition 4.1 and Proposition 4.5 below which provides the a priori bound $\|u(t)\|_{H^{2}} \leq$ $C\left\|u_{0}\right\|_{H^{2} \cap L^{1}}$ when $\left\|u_{0}\right\|_{H^{2} \cap L^{1}}$ is sufficiently small. 
We next consider the a priori estimates for $u(t)$. Let $r_{0}$ be a number satisfying $0<r_{0} \leq 1$. We introduce the cut-off function $\mathbf{1}_{\left\{|\xi| \leq r_{0}\right\}}$ defined by

$$
\mathbf{1}_{\left\{|\xi| \leq r_{0}\right\}}= \begin{cases}1 & \left(|\xi|<r_{0}\right) \\ 0 & \left(|\xi| \geq r_{0}\right) .\end{cases}
$$

We introduce the projections $P_{1}$ and $P_{\infty}$ defined by

$$
P_{1} u=\mathcal{F}^{-1} \mathbf{1}_{\left\{|\xi| \leq r_{0}\right\}} \Pi \mathcal{F} u, \quad P_{\infty}=I-P_{1} .
$$

It follows from Lemma 3.2 that

$$
\begin{aligned}
P_{1} e^{-t L} u_{0}= & \mathcal{F}^{-1} \mathbf{1}_{\left\{|\xi| \leq r_{0}\right\}}\left[e^{\lambda_{+, 0} t} P_{+, 0}+e^{\lambda_{-, 0} t} P_{-, 0}\right] \Pi \hat{u}_{0} \\
= & \mathcal{F}^{-1} \frac{\mathbf{1}_{\left\{|\xi| \leq r_{0}\right\}}}{\lambda_{+, 0}-\lambda_{-, 0}}\left[e^{\lambda_{+, 0} t}\left(\begin{array}{ccc}
-\lambda_{-, 0} & i \gamma \xi & 0 \\
i \gamma \xi & \lambda_{+, 0} & 0 \\
0 & 0 & 0
\end{array}\right)\right. \\
& \left.+e^{\lambda_{-, 0} t}\left(\begin{array}{ccc}
\lambda_{+, 0} & -i \gamma \xi & 0 \\
-i \gamma \xi & -\lambda_{-, 0} & 0 \\
0 & 0 & 0
\end{array}\right)\right]\left(\begin{array}{c}
\left\langle\hat{\phi}_{0}\right\rangle \\
\left\langle\hat{w}_{0}^{1}\right\rangle \\
0
\end{array}\right) .
\end{aligned}
$$

for $u_{0}={ }^{\top}\left(\phi_{0}, w_{0}^{1}, w_{0}^{2}\right)$. We also note that $P_{1} u$ does not depend on $x_{2}$, and so,

$$
\partial_{x_{2}} P_{1} u=0 .
$$

We decompose $u={ }^{\top}(\phi, w)$ into

$$
u=u_{1}+u_{\infty}
$$

where

$$
u_{1}=P_{1} u={ }^{\top}\left(\phi_{1}, w_{1}^{1}, w_{1}^{2}\right), \quad u_{\infty}=P_{\infty} u={ }^{\top}\left(\phi_{\infty}, w_{\infty}^{1}, w_{\infty}^{2}\right) .
$$

Remark 4.2 We see from the definition of $P_{1}$ that $u_{1}=u_{1}\left(x_{1}, t\right)$ satisfies

$$
\left\|\partial_{x_{1}}^{k+l} u_{1}\right\|_{2} \leq\left\|\partial_{x_{1}}^{l} u_{1}\right\|_{2}
$$

for arbitrary $k$ and $l$. We also note that $u_{\infty}$ satisfies

$$
\left\|u_{\infty}\right\|_{2} \leq C\left\|\partial_{x} u_{\infty}\right\|_{2}
$$

We will frequently make use of these properties in the subsequent arguments. 
Proposition 4.3 Let $u(t)$ be a solution of (4.1) on $[0, T]$. Assume that $u \in C\left([0, T] ; H^{2} \times H_{*}^{2}\right) \cap C^{1}\left([0, T] ; L^{2}\right)$ with $w \in L^{2}\left(0, T ; H^{3}\right)$. Then

$$
u_{1}={ }^{\top}\left(\phi_{1}, w_{1}\right) \in C^{1}\left([0, T] ; H^{l}(\Omega)\right) \quad(\forall l=0,1,2, \cdots)
$$

and

$$
u_{\infty}={ }^{\top}\left(\phi_{\infty}, w_{\infty}\right) \in C\left([0, T] ; H^{2} \times H_{*}^{2}\right) \cap C^{1}\left([0, T] ; L^{2}\right)
$$

with $w_{\infty} \in L^{2}\left(0, T ; H^{3}\right)$.

Furthermore, $u_{1}$ and $u_{\infty}$ satisfy

$$
\begin{gathered}
u_{1}=P_{1} e^{-t L} u_{0}+\int_{0}^{t} P_{1} e^{-(t-\tau) L} F(u(\tau)) \mathrm{d} \tau, \\
\partial_{t} u_{\infty}+L u_{\infty}=F_{\infty},\left.\quad u_{\infty}\right|_{t=0}=P_{\infty} u_{0},
\end{gathered}
$$

where $F_{\infty}=P_{\infty} F={ }^{\top}\left(f_{\infty}^{0}, \tilde{f}_{\infty}\right), \tilde{f}_{\infty}=\left(f_{\infty}^{1}, f_{\infty}^{2}\right)$.

Proof. Since $P_{j} L \subset L P_{j}(j=1, \infty)$, applying $P_{j}$ to (4.1) we obtain the desired results.

We define $M(t) \geq 0$ by

$$
M(t)=M_{1}(t)+M_{\infty}(t) \quad(t \in[0, T]) .
$$

Here $M_{1}(t)$ and $M_{\infty}(t)$ are define by

$$
\begin{aligned}
& M_{1}(t)=\sup _{0 \leq \tau \leq t}\left\{\sum_{k=0}^{2}(1+\tau)^{\frac{1}{4}+\frac{k}{2}}\left\|\partial_{x_{1}}^{k} u_{1}(\tau)\right\|_{2}+(1+\tau)^{\frac{3}{4}}\left\|\partial_{\tau} u_{1}(\tau)\right\|_{2}\right\}, \\
& M_{\infty}(t)=\left(\sup _{0 \leq \tau \leq t}(1+\tau)^{\frac{5}{2}}\left\{\left\|u_{\infty}(\tau)\right\|_{H^{2}}^{2}+\left\|\partial_{\tau} u_{\infty}(\tau)\right\|_{2}^{2}\right\}\right)^{\frac{1}{2}} .
\end{aligned}
$$

We note that, by the Gagliardo-Nirenberg-Sobolev inequality,

$$
\begin{aligned}
\left\|u_{1}(t)\right\|_{\infty} & \leq C\left\|u_{1}(t)\right\|_{2}^{\frac{1}{2}}\left\|\partial_{x_{1}} u_{1}(t)\right\|_{2}^{\frac{1}{2}} \leq C(1+t)^{-\frac{1}{2}} M_{1}(t), \\
\left\|u_{\infty}(t)\right\|_{\infty} & \leq C\left\|u_{\infty}(t)\right\|_{H^{2}} \leq C(1+t)^{-\frac{5}{4}} M_{\infty}(t) .
\end{aligned}
$$

We introduce the quantities $E_{\infty}(t)$ and $D_{\infty}(t)$ for $u_{\infty}(t)={ }^{\top}\left(\phi_{\infty}(t), w_{\infty}(t)\right)$ :

$$
\begin{aligned}
& E_{\infty}(t)=\left\|u_{\infty}(t)\right\|_{H^{2}}^{2}+\left\|\partial_{t} u_{\infty}(t)\right\|_{2}^{2}, \\
& D_{\infty}(t)=\left\|\nabla \phi_{\infty}(t)\right\|_{H^{1}}^{2}+\left\|\nabla w_{\infty}(t)\right\|_{H^{2}}^{2}+\left\|\partial_{t} u_{\infty}(t)\right\|_{H^{1}}^{2} .
\end{aligned}
$$


Proposition 4.4 Let $u(t)$ be a solution of (4.1) on $[0, T]$. Then there exists $\varepsilon_{1}>0$ such that if $\|u(t)\|_{H^{2}} \leq \varepsilon_{1}$ and $M(t) \leq 1$ for $t \in[0, T]$, the estimates

$$
M_{1}(t) \leq C\left\{\left\|u_{0}\right\|_{1}+M(t)^{2}\right\}
$$

and

$$
\begin{aligned}
& E_{\infty}(t)+\int_{0}^{t} e^{-a(t-\tau)} D_{\infty}(\tau) \mathrm{d} \tau \\
& \leq C\left\{e^{-a t} E_{\infty}(0)+(1+t)^{-\frac{5}{2}} M(t)^{4}+\int_{0}^{t} e^{-a(t-\tau)} \mathcal{R}(\tau) \mathrm{d} \tau\right\}
\end{aligned}
$$

hold uniformly for $t \in[0, T]$ with $C>0$ independent of $T$. Here $a=a(\nu, \tilde{\nu}, \gamma)$ is a positive constant; and $\mathcal{R}(t)$ is a function satisfying the estimate

$$
\mathcal{R}(t) \leq C\left\{(1+t)^{-\frac{5}{2}} M(t)^{3}+M(t) D_{\infty}(t)\right\}
$$

The estimate (4.9) will be proved in section 5, and the estimates (4.10) and (4.11) will be proved in sections 6 and 7 .

From Proposition 4.4, one can show the following uniform estimate of $M(t)$ as in [4].

Proposition 4.5 If $\left\|u_{0}\right\|_{H^{2} \cap L^{1}}$ is sufficiently small, then

$$
M(t) \leq C\left\|u_{0}\right\|_{H^{2} \cap L^{1}} .
$$

Theorem 2.1 now follows from Propositions 4.1 and 4.5.

\section{Estimates on $P_{1} u$}

In this section we estimate the low-frequency part $u_{1}=P_{1} u$ and prove estimate (4.9) in Proposition 4.4.

Proof of (4.9). We see from Lemma 3.2 and the definition of $\Pi$ that

$$
\begin{aligned}
\left\|\partial_{x_{1}}^{l} e^{-t L} P_{1} u_{0}\right\|_{2} & \leq C\left(\int_{\mathbb{R}}|\xi|^{2 l} e^{-c_{0}|\xi|^{2} t} \mathbf{1}_{\left\{|\xi| \leq r_{0}\right\}}\left|\Pi \hat{u}_{0}\right|^{2} d \xi\right)^{\frac{1}{2}} \\
& \leq C\left(\int_{\mathbb{R}}|\xi|^{2 l} e^{-c_{0}|\xi|^{2} t} \mathbf{1}_{\left\{|\xi| \leq r_{0}\right\}} d \xi\right)^{\frac{1}{2}}\left\|u_{0}\right\|_{1} \\
& \leq C(1+t)^{-\frac{1}{4}-\frac{l}{2}}\left\|u_{0}\right\|_{1}
\end{aligned}
$$


for $l \geq 0$, and hence, by (4.6), we have

$$
\begin{aligned}
\left\|\partial_{x_{1}}^{k} u_{1}(t)\right\|_{2} & \leq\left\|\partial_{x_{1}}^{k} e^{-t L} P_{1} u_{0}\right\|_{2}+\int_{0}^{t}\left\|\partial_{x_{1}}^{k} e^{-(t-\tau) L} P_{1} F(u(\tau))\right\|_{2} \mathrm{~d} \tau \\
& \leq C(1+t)^{-\frac{1}{4}-\frac{k}{2}}\left\|u_{0}\right\|_{1}+\int_{0}^{t}\left\|\partial_{x_{1}}^{k} e^{-(t-\tau) L} P_{1} F(u(\tau))\right\|_{2} \mathrm{~d} \tau
\end{aligned}
$$

for $k=0,1,2$.

Let us estimate $\int_{0}^{t}\left\|\partial_{x_{1}}^{k} e^{-(t-\tau) L} P_{1} F(u(\tau))\right\|_{2} \mathrm{~d} \tau$. By the Sobolev inequality: $\|\phi\|_{\infty} \leq C\|\phi\|_{H^{2}}$, we see that there exists $\varepsilon_{2}>0$ such that if $\|u(t)\|_{H^{2}} \leq \varepsilon_{2}$ for $t \in[0, T]$, then $\|\phi(t)\|_{\infty} \leq \frac{1}{2}$ for $t \in[0, T]$, and hence $P_{1} F(u)$ is written as

$$
P_{1} F(u)=P_{1} \partial_{x_{1}} F_{0}(u)+P_{1} \tilde{F}(u)
$$

where

$$
F_{0}(u)=\left(\begin{array}{c}
-\gamma \phi_{1} w_{1}^{1} \\
-\frac{\gamma}{2}\left(w_{1}^{1}\right)^{2}+(\nu+\tilde{\nu})\left(\phi_{1} \partial_{x_{1}} w_{1}^{1}\right)+\frac{\gamma}{2} \phi_{1}^{2}-\frac{\rho_{*}}{\gamma} P^{(2)}(\phi) \phi_{1}^{2} \\
0
\end{array}\right)
$$

Here each term in $P_{1} \tilde{F}(u)$ includes $u_{\infty}, O\left(\left(\partial_{x_{1}} u_{1}\right)^{2}\right), O\left(u_{1}^{2} \partial_{x_{1}} \phi_{1}\right)$ or $O\left(u_{1}^{2} \partial_{x_{1}}^{l} w_{1}\right) \quad(l=$ $1,2)$, and $P_{1} \tilde{F}(u)$ is estimated as

$$
\left\|P_{1} \tilde{F}(u(\tau))\right\|_{1} \leq C(1+\tau)^{-\frac{5}{4}} M(t)^{2} .
$$

It then follows from (5.1) that

$$
\begin{aligned}
\int_{0}^{t}\left\|\partial_{x_{1}}^{k} e^{-(t-\tau) L} P_{1} \tilde{F}(u(\tau))\right\|_{2} \mathrm{~d} \tau & \leq C \int_{0}^{t}(1+t-\tau)^{-\frac{1}{4}-\frac{k}{2}}(1+\tau)^{-\frac{5}{4}} \mathrm{~d} \tau M(t)^{2} \\
& \leq C(1+t)^{-\frac{1}{4}-\frac{k}{2}} M(t)^{2}
\end{aligned}
$$

As for the estimates for $P_{1} \partial_{x_{1}} F_{0}(u)$ part, we write it as

$$
\begin{aligned}
& \int_{0}^{t}\left\|\partial_{x_{1}}^{k} e^{-(t-\tau) L} P_{1} \partial_{x_{1}} F_{0}(u(\tau))\right\|_{2} \mathrm{~d} \tau \\
& =\left(\int_{0}^{\frac{t}{2}}+\int_{\frac{t}{2}}^{t}\right)\left\|\partial_{x_{1}}^{k} e^{-(t-\tau) L} P_{1} \partial_{x_{1}} F_{0}(u(\tau))\right\|_{2} \mathrm{~d} \tau \\
& =: I_{1}+I_{2} .
\end{aligned}
$$


Since $\partial_{x_{1}} e^{-t L} P_{1}=e^{-t L} P_{1} \partial_{x_{1}}$, we have

$$
\begin{aligned}
I_{1} & =\int_{0}^{\frac{t}{2}}\left\|\partial_{x_{1}}^{k+1} e^{-(t-\tau) L} P_{1} F_{0}(u(\tau))\right\|_{2} \mathrm{~d} \tau \\
& \leq C \int_{0}^{\frac{t}{2}}(1+t-\tau)^{-\frac{3}{4}-\frac{k}{2}}(1+\tau)^{-\frac{1}{2}} \mathrm{~d} \tau M(t)^{2} \\
& \leq C(1+t)^{-\frac{1}{4}-\frac{k}{2}} M(t)^{2}, \quad k=0,1,2 .
\end{aligned}
$$

As for $I_{2}$, we have, for $k=0,1$,

$$
\begin{aligned}
I_{2} & \leq C \int_{\frac{t}{2}}^{t}(1+t-\tau)^{-\frac{1}{4}-\frac{k}{2}}(1+\tau)^{-1} \mathrm{~d} \tau M(t)^{2} \\
& \leq C(1+t)^{-\frac{1}{4}-\frac{k}{2}} M(t)^{2} .
\end{aligned}
$$

For $k=2$, we have

$$
\begin{aligned}
I_{2} & \leq C \int_{\frac{t}{2}}^{t}\left\|\partial_{x_{1}} e^{-(t-\tau) L} P_{1} \partial_{x_{1}}^{2} F_{0}(u(\tau))\right\|_{2} \mathrm{~d} \tau \\
& \leq C \int_{\frac{t}{2}}^{t}(1+t-\tau)^{-\frac{3}{4}}(1+\tau)^{-\frac{3}{2}} \mathrm{~d} \tau M(t)^{2} \\
& \leq C(1+t)^{-\frac{5}{4}} M(t)^{2} .
\end{aligned}
$$

We thus obtain

$$
\left\|\partial_{x_{1}}^{k} u_{1}(t)\right\|_{2} \leq C(1+t)^{-\frac{1}{4}-\frac{k}{2}}\left\{\left\|u_{0}\right\|_{1}+M(t)^{2}\right\} .
$$

We next estimate the time derivative. We have

$$
\begin{aligned}
\left\|-L u_{1}(t)\right\|_{2} & \leq C\left\{\left\|\partial_{x_{1}}^{2} w_{1}(t)\right\|_{2}+\left\|\partial_{x_{1}} u_{1}(t)\right\|_{2}\right\} \\
& \leq C(1+t)^{-\frac{3}{4}}\left\{\left\|u_{0}\right\|_{1}+M(t)^{2}\right\}
\end{aligned}
$$

and

$$
\left\|P_{1} F(u(t))\right\|_{2} \leq C(1+t)^{-\frac{5}{4}} M(t)^{2} .
$$

Since

$$
\partial_{t} u_{1}=-L u_{1}+P_{1} F(u)
$$

we obtain

$$
\begin{aligned}
\left\|\partial_{t} u_{1}(t)\right\|_{2} & \leq\left\|L u_{1}(t)\right\|_{2}+\left\|P_{1} F(u(t))\right\|_{2} \\
& \leq C(1+t)^{-\frac{3}{4}}\left\{\left\|u_{0}\right\|_{1}+M(t)^{2}\right\} .
\end{aligned}
$$

By (5.2) and (5.3), we deduce the desired estimate. This completes the proof. 


\section{Estimates on $P_{\infty} u$}

In this section we estimate the high-frequency part $u_{\infty}=P_{\infty} u$ by using the Matsumura-Nishida energy method to prove estimate (4.10) in Proposition 4.4 .

We introduce the quantity $D[w]$ which is defined by

$$
D[w]=\nu\|\nabla w\|_{2}^{2}+\tilde{\nu}\|\operatorname{div} w\|_{2}^{2}
$$

We also define operators $\tilde{P}_{1}$ and $\tilde{P}_{\infty}$ by

$$
\tilde{P}_{1} \phi=\mathcal{F}^{-1} \mathbf{1}_{\left\{|\xi| \leq r_{0}\right\}}\langle\mathcal{F} \phi\rangle, \quad \tilde{P}_{\infty}=I-\tilde{P}_{1}
$$

Note that $P_{1} u={ }^{\top}\left(\tilde{P}_{1} \phi, \tilde{P}_{1} w^{1}, 0\right)$ and $P_{\infty} u={ }^{\top}\left(\tilde{P}_{\infty} \phi, \tilde{P}_{\infty} w^{1}, w^{2}\right)$ for $u=$ ${ }^{\top}\left(\phi, w^{1}, w^{2}\right)$.

To prove (4.10), we prepare some basic estimates.

Proposition 6.1 Let $k$ and $j$ be nonnegative integers satisfying $0 \leq 2 k+j \leq$ 2. Then

$$
\frac{1}{2} \frac{\mathrm{d}}{\mathrm{d} t}\left\|\partial_{t}^{k} \partial_{x_{1}}^{j} u_{\infty}\right\|_{2}^{2}+D\left[\partial_{t}^{k} \partial_{x_{1}}^{j} w_{\infty}\right]+\frac{\nu+\tilde{\nu}}{\gamma^{2}}\left\|\partial_{t}^{k} \partial_{x_{1}}^{j} \dot{\phi}_{\infty}\right\|_{2}^{2} \leq C R_{j, k}^{(1)},
$$

where

$$
\begin{aligned}
\dot{\phi}_{\infty}= & \partial_{t} \phi_{\infty}+\gamma w \cdot \nabla \phi_{\infty} \\
R_{j, k}^{(1)}= & \frac{\gamma}{2}\left(\operatorname{div} w,\left|\partial_{t}^{k} \partial_{x_{1}}^{j} \phi_{\infty}\right|^{2}\right)-\gamma\left(\left[\partial_{t}^{k} \partial_{x_{1}}^{j}, w\right] \cdot \nabla \phi_{\infty}, \partial_{t}^{k} \partial_{x_{1}}^{j} \phi_{\infty}\right) \\
& +\left(\partial_{t}^{k} \partial_{x_{1}}^{j} \tilde{f}_{\infty}^{0}, \partial_{t}^{k} \partial_{x_{1}}^{j} \phi_{\infty}\right)+\left(\partial_{t}^{k} \partial_{x_{1}}^{j} \tilde{f}_{\infty}, \partial_{t}^{k} \partial_{x_{1}}^{j} w_{\infty}\right)+\frac{\nu+\tilde{\nu}}{\gamma^{2}}\left\|\partial_{t}^{k} \partial_{x_{1}}^{j} \tilde{f}_{\infty}^{0}\right\|_{2}^{2} .
\end{aligned}
$$

Here and in what follows, $\tilde{f}_{\infty}^{0}$ denotes

$$
\tilde{f}_{\infty}^{0}=\gamma \tilde{P}_{1}\left(w \cdot \nabla \phi_{\infty}\right)-\gamma \tilde{P}_{\infty}\left(w \cdot \nabla \phi_{1}+\phi \operatorname{div} w\right) .
$$

Proof. Equation (4.7) is written as

$$
\begin{gathered}
\partial_{t} \phi_{\infty}+\gamma w \cdot \nabla \phi_{\infty}+\gamma \operatorname{div} w_{\infty}=\tilde{f}_{\infty}^{0}, \\
\partial_{t} w_{\infty}-\nu \Delta w_{\infty}-\tilde{\nu} \nabla \operatorname{div} w_{\infty}+\gamma \nabla \phi_{\infty}=\tilde{f}_{\infty} .
\end{gathered}
$$

We compute $\left(\partial_{x_{1}}^{j}(6.2), \partial_{x_{1}}^{j} \phi_{\infty}\right)+\left(\partial_{x_{1}}^{j}(6.3), \partial_{x_{1}}^{j} w_{\infty}\right)$ to obtain

$$
\begin{aligned}
\frac{1}{2} & \frac{\mathrm{d}}{\mathrm{d} t}\left\|\partial_{x_{1}}^{j} u_{\infty}\right\|_{2}^{2}+D\left[\partial_{x_{1}}^{j} w_{\infty}\right] \\
= & -\gamma\left(w \cdot \nabla \partial_{x_{1}}^{j} \phi_{\infty}, \partial_{x_{1}}^{j} \phi_{\infty}\right)-\gamma\left(\left[\partial_{x_{1}}^{j}, w\right] \cdot \nabla \phi_{\infty}, \partial_{x_{1}}^{j} \phi_{\infty}\right) \\
& +\left(\partial_{x_{1}}^{j} \tilde{f}_{\infty}^{0}, \partial_{x_{1}}^{j} \phi_{\infty}\right)+\left(\partial_{x_{1}}^{j} \tilde{f}_{\infty}, \partial_{x_{1}}^{j} w_{\infty}\right) \\
= & \frac{\gamma}{2}\left(\operatorname{div} w,\left|\partial_{x_{1}}^{j} \phi_{\infty}\right|^{2}\right)-\gamma\left(\left[\partial_{x_{1}}^{j}, w\right] \cdot \nabla \phi_{\infty}, \partial_{x_{1}}^{j} \phi_{\infty}\right) \\
& +\left(\partial_{x_{1}}^{j} \tilde{f}_{\infty}^{0}, \partial_{x_{1}}^{j} \phi_{\infty}\right)+\left(\partial_{x_{1}}^{j} \tilde{f}_{\infty}, \partial_{x_{1}}^{j} w_{\infty}\right) .
\end{aligned}
$$


We set $\dot{\phi}:=\partial_{t} \phi+\gamma w \cdot \nabla \phi$. From (6.2), we have $\partial_{x_{1}}^{j} \dot{\phi}_{\infty}=-\gamma \operatorname{div} \partial_{x_{1}}^{j} w_{\infty}+\partial_{x_{1}}^{j} \tilde{f}_{\infty}^{0}$, and hence

$$
\left\|\partial_{x_{1}}^{j} \dot{\phi}_{\infty}\right\|_{2}^{2} \leq C\left(\gamma^{2}\left\|\operatorname{div} \partial_{x_{1}}^{j} w_{\infty}\right\|_{2}^{2}+\left\|\partial_{x_{1}}^{j} \tilde{f}_{\infty}^{0}\right\|_{2}^{2}\right) .
$$

We thus obtain

$$
\begin{aligned}
& \frac{\nu+\tilde{\nu}}{\gamma^{2}}\left\|\partial_{x_{1}}^{j} \dot{\phi}_{\infty}\right\|_{2}^{2} \\
& \leq C\left\{\nu\left\|\nabla \partial_{x_{1}}^{j} w_{\infty}\right\|_{2}^{2}+\tilde{\nu}\left\|\operatorname{div} \partial_{x_{1}}^{j} w_{\infty}\right\|_{2}^{2}+\frac{\nu+\tilde{\nu}}{\gamma^{2}}\left\|\partial_{x_{1}}^{j} \tilde{f}_{\infty}^{0}\right\|_{2}^{2}\right\} \\
& =C\left\{D\left[\partial_{x_{1}}^{j} w_{\infty}\right]+\frac{\nu+\tilde{\nu}}{\gamma^{2}}\left\|\partial_{x_{1}}^{j} \tilde{f}_{\infty}^{0}\right\|_{2}^{2}\right\} .
\end{aligned}
$$

By $(6.4)+\frac{1}{2 C} \times(6.5)$, we obtain

$$
\frac{\mathrm{d}}{\mathrm{d} t}\left\|\partial_{x_{1}}^{j} u_{\infty}\right\|_{2}^{2}+D\left[\partial_{x_{1}}^{j} w_{\infty}\right]+\frac{\nu+\tilde{\nu}}{C \gamma^{2}}\left\|\partial_{x_{1}}^{j} \dot{\phi}_{\infty}\right\|_{2}^{2} \leq C R_{j, 0}^{(1)}
$$

Replacing $\partial_{x_{1}}^{j}$ by $\partial_{t}$, we also have

$$
\frac{\mathrm{d}}{\mathrm{d} t}\left\|\partial_{t} u_{\infty}\right\|_{2}^{2}+D\left[\partial_{t} w_{\infty}\right]+\frac{\nu+\tilde{\nu}}{C \gamma^{2}}\left\|\partial_{t} \dot{\phi}_{\infty}\right\|_{2}^{2} \leq C R_{0,1}^{(1)}
$$

This completes the proof.

Proposition 6.2 It holds that

$$
\begin{aligned}
& \frac{1}{2} \frac{\mathrm{d}}{\mathrm{d} t}\left\{D\left[w_{\infty}\right]-2 \gamma\left(\phi_{\infty}, \operatorname{div} w_{\infty}\right)\right\}+\frac{1}{2}\left\|\partial_{t} u_{\infty}\right\|_{2}^{2} \\
& \leq C\left\{\gamma^{2}\left\|\operatorname{div} w_{\infty}\right\|_{2}^{2}+\left\|\tilde{f}_{\infty}^{0}\right\|_{2}^{2}+\left\|\tilde{f}_{\infty}\right\|_{2}^{2}+\left\|w \cdot \nabla \phi_{\infty}\right\|_{2}^{2}\right\}
\end{aligned}
$$

Proof. We compute $\left((6.2), \partial_{t} \phi_{\infty}\right)+\left((6.3), \partial_{t} w_{\infty}\right)$ to obtain

$$
\begin{aligned}
& \left\|\partial_{t} u_{\infty}\right\|_{2}^{2}+\frac{1}{2} \frac{\mathrm{d}}{\mathrm{d} t} D\left[w_{\infty}\right]+\gamma\left\{\left(\operatorname{div} w_{\infty}, \partial_{t} \phi_{\infty}\right)+\left(\nabla \phi_{\infty}, \partial_{t} w_{\infty}\right)\right\} \\
& =-\gamma\left(\left(w \cdot \nabla \phi_{\infty}\right), \partial_{t} \phi_{\infty}\right)+\left(\tilde{f}_{\infty}^{0}, \partial_{t} \phi_{\infty}\right)+\left(\tilde{f}_{\infty}, \partial_{t} w_{\infty}\right) .
\end{aligned}
$$

Since $\left(\nabla \phi_{\infty}, \partial_{t} w_{\infty}\right)=-\left(\phi_{\infty}, \operatorname{div} \partial_{t} w_{\infty}\right)$, we have

$$
\begin{aligned}
& \left\|\partial_{t} u_{\infty}\right\|_{2}^{2}+\frac{1}{2} \frac{\mathrm{d}}{\mathrm{d} t} D\left[w_{\infty}\right]+\gamma\left\{\left(\operatorname{div} w_{\infty}, \partial_{t} \phi_{\infty}\right)-\left(\phi_{\infty}, \operatorname{div} \partial_{t} w_{\infty}\right)\right\} \\
& =-\gamma\left(w \cdot \nabla \phi_{\infty}, \partial_{t} \phi_{\infty}\right)+\left(\tilde{f}_{\infty}^{0}, \partial_{t} \phi_{\infty}\right)+\left(\tilde{f}_{\infty}, \partial_{t} w_{\infty}\right) \\
& \leq \frac{1}{4}\left\|\partial_{t} u_{\infty}\right\|_{2}^{2}+C\left\{\left\|w \cdot \nabla \phi_{\infty}\right\|_{2}^{2}+\left\|\tilde{f}_{\infty}^{0}\right\|_{2}^{2}+\left\|\tilde{f}_{\infty}\right\|_{2}^{2}\right\}
\end{aligned}
$$


Adding $-2 \gamma\left(\operatorname{div} w_{\infty}, \partial_{t} \phi_{\infty}\right)$ to both sides of (6.9), we obtain

$$
\begin{aligned}
& \left\|\partial_{t} u_{\infty}\right\|_{2}^{2}+\frac{1}{2} \frac{\mathrm{d}}{\mathrm{d} t} D\left[w_{\infty}\right]-\gamma \frac{\mathrm{d}}{\mathrm{d} t}\left(\phi_{\infty}, \operatorname{div} w_{\infty}\right) \\
& \leq-2 \gamma\left(\operatorname{div} w_{\infty}, \partial_{t} \phi_{\infty}\right)+\frac{1}{4}\left\|\partial_{t} u_{\infty}\right\|_{2}^{2}+C\left\{\left\|w \cdot \nabla \phi_{\infty}\right\|_{2}^{2}+\left\|\tilde{f}_{\infty}^{0}\right\|_{2}^{2}+\left\|\tilde{f}_{\infty}\right\|_{2}^{2}\right\} \\
& \leq \frac{1}{2}\left\|\partial_{t} u_{\infty}\right\|_{2}^{2}+C\left\{\gamma^{2}\left\|\operatorname{div} w_{\infty}\right\|_{2}^{2}+\left\|w \cdot \nabla \phi_{\infty}\right\|_{2}^{2}+\left\|\tilde{f}_{\infty}^{0}\right\|_{2}^{2}+\left\|\tilde{f}_{\infty}\right\|_{2}^{2}\right\},
\end{aligned}
$$

which gives the desired estimate. This completes the proof.

Proposition 6.3 Let $j$ and $l$ be integers satisfying $0 \leq j+l \leq 1$. Then

$$
\begin{aligned}
& \frac{1}{2} \frac{\mathrm{d}}{\mathrm{d} t}\left\|\partial_{x_{1}}^{j} \partial_{x_{2}}^{l+1} \phi_{\infty}\right\|_{2}^{2}+\frac{\gamma^{2}}{\nu+\tilde{\nu}}\left\|\partial_{x_{1}}^{j} \partial_{x_{2}}^{l+1} \phi_{\infty}\right\|_{2}^{2} \\
& \leq C R_{j, l}^{(2)}+C\left\{\frac{1}{\nu+\tilde{\nu}}\left\|\partial_{t} \partial_{x_{1}}^{j} \partial_{x_{2}}^{l} w_{\infty}\right\|_{2}^{2}+\frac{\nu^{2}}{\nu+\tilde{\nu}}\left\|\partial_{x_{1}}^{j+1} \partial_{x_{2}}^{l} \nabla w_{\infty}\right\|_{2}^{2}\right\},
\end{aligned}
$$

where

$$
\begin{aligned}
R_{j, l}^{(2)}= & \frac{\gamma}{2}\left(\operatorname{div} w,\left|\partial_{x_{1}}^{j} \partial_{x_{2}}^{l+1} \phi_{\infty}\right|^{2}\right)+C\left\{(\nu+\tilde{\nu})\left\|\left[\partial_{x_{1}}^{j} \partial_{x_{2}}^{l+1}, w\right] \cdot \nabla \phi_{\infty}\right\|_{2}^{2}\right. \\
& \left.+\frac{\nu+\tilde{\nu}}{\gamma^{2}}\left\|\partial_{x_{1}}^{j} \partial_{x_{2}}^{l} h_{\infty}^{0}\right\|_{2}^{2}\right\} \\
h_{\infty}^{0}= & \partial_{x_{2}} \tilde{f}_{\infty}^{0}+\frac{\gamma}{\nu+\tilde{\nu}} f_{\infty}^{2} .
\end{aligned}
$$

Proof. We compute $\partial_{x_{2}}(6.2)+\frac{\gamma}{\nu+\tilde{\nu}} \times($ the second component of $(6.3))$ to obtain

$$
\begin{aligned}
& \partial_{x_{2}} \dot{\phi}_{\infty}+\gamma \partial_{x_{2}} \operatorname{div} w_{\infty}+\frac{\gamma}{\nu+\tilde{\nu}} \partial_{t} w_{\infty}^{2}-\frac{\nu \gamma}{\nu+\tilde{\nu}} \Delta w_{\infty}^{2} \\
& -\frac{\tilde{\nu} \gamma}{\nu+\tilde{\nu}} \partial_{x_{2}} \operatorname{div} w_{\infty}+\frac{\gamma^{2}}{\nu+\tilde{\nu}} \partial_{x_{2}} \phi_{\infty}=h_{\infty}^{0} .
\end{aligned}
$$

This gives

$$
\partial_{x_{2}} \dot{\phi}_{\infty}+\frac{\gamma^{2}}{\nu+\tilde{\nu}} \partial_{x_{2}} \phi_{\infty}=h_{\infty}^{0}-\frac{\gamma}{\nu+\tilde{\nu}} H\left(w_{\infty}\right)
$$

where

$$
H(w)=\partial_{t} w^{2}-\nu \partial_{x_{1}}^{2} w^{2}+\nu \partial_{x_{1}} \partial_{x_{2}} w^{1} .
$$

Applying $\partial_{x_{1}}^{j} \partial_{x_{2}}^{l}$ to (6.11) we have

$$
\partial_{x_{1}}^{j} \partial_{x_{2}}^{l+1} \dot{\phi}_{\infty}+\frac{\gamma^{2}}{\nu+\tilde{\nu}} \partial_{x_{1}}^{j} \partial_{x_{2}}^{l+1} \phi_{\infty}=\partial_{x_{1}}^{j} \partial_{x_{2}}^{l} h_{\infty}^{0}-\frac{\gamma}{\nu+\tilde{\nu}} H\left(\partial_{x_{1}}^{j} \partial_{x_{2}}^{l} w_{\infty}\right) .
$$


We also write (6.12) as

$$
\begin{aligned}
& \partial_{t} \partial_{x_{1}}^{j} \partial_{x_{2}}^{l+1} \phi_{\infty}+\frac{\gamma^{2}}{\nu+\tilde{\nu}} \partial_{x_{1}}^{j} \partial_{x_{2}}^{l+1} \phi_{\infty} \\
& =-\gamma w \cdot \nabla \partial_{x_{1}}^{j} \partial_{x_{2}}^{l+1} \phi_{\infty}-\gamma\left[\partial_{x_{1}}^{j} \partial_{x_{2}}^{l+1}, w\right] \cdot \nabla \phi_{\infty}+\partial_{x_{1}}^{j} \partial_{x_{2}}^{l} h_{\infty}^{0} \\
& \quad-\frac{\gamma}{\nu+\tilde{\nu}} H\left(\partial_{x_{1}}^{j} \partial_{x_{2}}^{l} w_{\infty}\right) .
\end{aligned}
$$

Taking the inner product of (6.13) with $\partial_{x_{1}}^{j} \partial_{x_{2}}^{l+1} \phi_{\infty}$ we have

$$
\begin{aligned}
\frac{1}{2} \frac{\mathrm{d}}{\mathrm{d} t}\left\|\partial_{x_{1}}^{j} \partial_{x_{2}}^{l+1} \phi_{\infty}\right\|_{2}^{2}+\frac{\gamma^{2}}{\nu+\tilde{\nu}}\left\|\partial_{x_{1}}^{j} \partial_{x_{2}}^{l+1} \phi_{\infty}\right\|_{2}^{2} \\
=\frac{\gamma}{2}\left(\operatorname{div} w,\left|\partial_{x_{1}}^{j} \partial_{x_{2}}^{l+1} \phi_{\infty}\right|^{2}\right)+\left(-\gamma\left[\partial_{x_{1}}^{j} \partial_{x_{2}}^{l+1}, w\right] \cdot \nabla \phi_{\infty}\right. \\
\left.\quad+\partial_{x_{1}}^{j} \partial_{x_{2}}^{l} h_{\infty}^{0}-\frac{\gamma}{\nu+\tilde{\nu}} H\left(\partial_{x_{1}}^{j} \partial_{x_{2}}^{l} w_{\infty}\right), \partial_{x_{1}}^{j} \partial_{x_{2}}^{l+1} \phi_{\infty}\right) \\
\leq \frac{\gamma^{2}}{2(\nu+\tilde{\nu})}\left\|\partial_{x_{1}}^{j} \partial_{x_{2}}^{l+1} \phi_{\infty}\right\|_{2}^{2}+\frac{\gamma}{2}\left(\operatorname{div} w,\left|\partial_{x_{1}}^{j} \partial_{x_{2}}^{l+1} \phi_{\infty}\right|^{2}\right) \\
\quad+C\left\{(\nu+\tilde{\nu})\left\|\left[\partial_{x_{1}}^{j} \partial_{x_{2}}^{l+1}, w\right] \cdot \nabla \phi_{\infty}\right\|_{2}^{2}+\frac{\nu+\tilde{\nu}}{\gamma^{2}}\left\|\partial_{x_{1}}^{j} \partial_{x_{2}}^{l} h_{\infty}^{0}\right\|_{2}^{2}\right. \\
\left.\quad+\frac{1}{\nu+\tilde{\nu}}\left\|\partial_{x_{1}}^{j} \partial_{x_{2}}^{l} \partial_{t} w_{\infty}\right\|_{2}^{2}+\frac{\nu^{2}}{\nu+\tilde{\nu}}\left\|\partial_{x_{1}}^{j+1} \partial_{x_{2}}^{l} \nabla w_{\infty}\right\|_{2}^{2}\right\} \\
=\frac{\gamma^{2}}{2(\nu+\tilde{\nu})}\left\|\partial_{x_{1}}^{j} \partial_{x_{2}}^{l+1} \phi_{\infty}\right\|_{2}^{2}+R_{j, l}^{(2)} \\
\quad+C\left\{\frac{1}{\nu+\tilde{\nu}}\left\|\partial_{x_{1}}^{j} \partial_{x_{2}}^{l} \partial_{t} w_{\infty}\right\|_{2}^{2}+\frac{\nu^{2}}{\nu+\tilde{\nu}}\left\|\partial_{x_{1}}^{j+1} \partial_{x_{2}}^{l} \nabla w_{\infty}\right\|_{2}^{2}\right\} .
\end{aligned}
$$

The desired estimate follows from (6.14). This completes the proof.

Proposition 6.4 Let $j$ and $l$ be integers satisfying $0 \leq j+l \leq 1$. Then

$$
\begin{aligned}
& \frac{1}{2} \frac{\mathrm{d}}{\mathrm{d} t}\left\|\partial_{x_{1}}^{j} \partial_{x_{2}}^{l+1} \phi_{\infty}\right\|_{2}^{2}+\frac{\gamma^{2}}{\nu+\tilde{\nu}}\left\|\partial_{x_{1}}^{j} \partial_{x_{2}}^{l+1} \phi_{\infty}\right\|_{2}^{2}+\frac{\nu+\tilde{\nu}}{\gamma^{2}}\left\|\partial_{x_{1}}^{j} \partial_{x_{2}}^{l+1} \dot{\phi}_{\infty}\right\|_{2}^{2} \\
& \leq C R_{j, l}^{(2)}+C\left\{\frac{1}{\nu+\tilde{\nu}}\left\|\partial_{t} \partial_{x_{1}}^{j} \partial_{x_{2}}^{l} w_{\infty}\right\|_{2}^{2}+\frac{\nu^{2}}{\nu+\tilde{\nu}}\left\|\partial_{x_{1}}^{j+1} \partial_{x_{2}}^{l} \nabla w_{\infty}\right\|_{2}^{2}\right\} .
\end{aligned}
$$

Proof. From (6.12), we get

$$
\begin{aligned}
& \frac{\nu+\tilde{\nu}}{\gamma^{2}}\left\|\partial_{x_{1}}^{j} \partial_{x_{2}}^{l+1} \dot{\phi}_{\infty}\right\|_{2}^{2} \\
& \leq C\left\{\frac{\gamma^{2}}{\nu+\tilde{\nu}}\left\|\partial_{x_{1}}^{j} \partial_{x_{2}}^{l+1} \phi_{\infty}\right\|_{2}^{2}+\frac{1}{\nu+\tilde{\nu}}\left\|\partial_{x_{1}}^{j} \partial_{x_{2}}^{l} \partial_{t} w_{\infty}\right\|_{2}^{2}\right. \\
& \left.\quad+\frac{\nu^{2}}{\nu+\tilde{\nu}}\left\|\partial_{x_{1}}^{j+1} \partial_{x_{2}}^{l} \nabla w_{\infty}\right\|_{2}^{2}+\frac{\nu+\tilde{\nu}}{\gamma^{2}}\left\|\partial_{x_{1}}^{j} \partial_{x_{2}}^{l} h_{\infty}^{0}\right\|_{2}^{2}\right\} .
\end{aligned}
$$


By $(6.10)+\frac{1}{2 C} \times(6.16)$, we obtain

$$
\begin{aligned}
& \frac{\mathrm{d}}{\mathrm{d} t}\left\|\partial_{x_{1}}^{j} \partial_{x_{2}}^{l+1} \phi_{\infty}\right\|_{2}^{2}+\frac{\gamma^{2}}{2(\nu+\tilde{\nu})}\left\|\partial_{x_{1}}^{j} \partial_{x_{2}}^{l+1} \phi_{\infty}\right\|_{2}^{2}+\frac{\nu+\tilde{\nu}}{2 C \gamma^{2}}\left\|\partial_{x_{1}}^{j} \partial_{x_{2}}^{l+1} \dot{\phi}_{\infty}\right\|_{2}^{2} \\
& \leq C R_{j, l}^{(2)}+C\left\{\frac{1}{\nu+\tilde{\nu}}\left\|\partial_{x_{1}}^{j} \partial_{x_{2}}^{l} \partial_{t} w_{\infty}\right\|_{2}^{2}+\frac{\nu^{2}}{\nu+\tilde{\nu}}\left\|\nabla \partial_{x_{1}}^{j+1} \partial_{x_{2}}^{l} w_{\infty}\right\|_{2}^{2}\right\} .
\end{aligned}
$$

This completes the proof.

Proposition 6.5 Let $j$ and $l$ be integers satisfying $0 \leq j+l \leq 1$. Then

$$
\begin{gathered}
\frac{\gamma^{2}}{\nu+\tilde{\nu}}\left\|\partial_{x_{1}}^{j} \partial_{x}^{l+1} \phi_{\infty}\right\|_{2}^{2}+\frac{\nu^{2}}{\nu+\tilde{\nu}}\left\|\partial_{x_{1}}^{j} \partial_{x}^{l+2} w_{\infty}\right\|_{2}^{2} \\
\leq C\left\{\frac{1}{\nu+\tilde{\nu}}\left\|\partial_{t} \partial_{x_{1}}^{j} w_{\infty}\right\|_{H^{l}}^{2}+\frac{\nu+\tilde{\nu}}{\gamma^{2}}\left\|\partial_{x_{1}}^{j} \dot{\phi}\right\|_{H^{l+1}}^{2}\right. \\
\left.+\frac{\nu+\tilde{\nu}}{\gamma^{2}}\left\|\partial_{x_{1}}^{j} \tilde{f}_{\infty}^{0}\right\|_{H^{l+1}}^{2}+\frac{1}{\nu+\tilde{\nu}}\left\|\partial_{x_{1}}^{j} \tilde{f}_{\infty}\right\|_{H^{l}}^{2}\right\}
\end{gathered}
$$

To prove Proposition 6.5 we will apply the following lemma.

Lemma 6.6 Let $u={ }^{\top}(p, v)$ be a solution of the Stokes system

$$
\left\{\begin{array}{l}
\operatorname{div} v=f \\
-\Delta v+\nabla p=g \\
\left.\partial_{x_{2}} v^{1}\right|_{x_{2}=0,1}=\left.v^{2}\right|_{x_{2}=0,1}=0
\end{array}\right.
$$

Then there exists a constant $C>0$ such that, for $0 \leq j+l \leq 1$,

$$
\left\|\partial_{x_{1}}^{j} \partial_{x}^{l+2} v\right\|_{2}+\left\|\partial_{x_{1}}^{j} \partial_{x}^{l+1} p\right\|_{2} \leq C\left\{\left\|\partial_{x_{1}}^{j} \partial_{x}^{l+1} f\right\|_{2}+\left\|\partial_{x_{1}}^{j} \partial_{x}^{l} g\right\|_{2}\right\} .
$$

It is not difficult to prove Lemma 6.6 by using the Fourier transform in $x_{1}$ and the Fourier series expansion in $x_{2}$ as in (3.8) and (3.9). More precisely, one can prove Lemma 6.6 for $l=0$ by using Lemma 3.2 with $\nu=\gamma=1, \tilde{\nu}=0$; and for $l=1$, in addition to Lemma 3.2, we also use the equations (6.19) to estimate $\left\|\partial_{x_{2}}^{2} p\right\|_{2}$ and $\left\|\partial_{x_{2}}^{3} v\right\|_{2}$. We omit the detail.

Proof of Proposition 6.5. We rewrite equation (6.2)-(6.3) in the following form:

$$
\left\{\begin{array}{l}
\operatorname{div} w_{\infty}=\frac{1}{\gamma}\left(\tilde{f}_{\infty}^{0}-\dot{\phi}_{\infty}\right) \\
-\Delta w_{\infty}+\nabla\left(\frac{\gamma}{\nu} \phi_{\infty}\right)=\frac{1}{\nu}\left\{\tilde{f}_{\infty}-\left(\partial_{t} w_{\infty}-\frac{\tilde{\nu}}{\gamma} \nabla\left(\tilde{f}_{\infty}^{0}-\dot{\phi}_{\infty}\right)\right\}\right.
\end{array}\right.
$$


It then follows from Lemma 6.6 that

$$
\begin{aligned}
& \frac{\gamma^{2}}{\nu^{2}}\left\|\partial_{x_{1}}^{j} \partial_{x}^{l+1} \phi_{\infty}\right\|_{2}^{2}+\left\|\partial_{x_{1}}^{j} \partial_{x}^{l+2} w_{\infty}\right\|_{2}^{2} \\
& \leq C\left\{\left\|\frac{1}{\gamma} \partial_{x_{1}}^{j}\left(\tilde{f}_{\infty}^{0}-\dot{\phi}_{\infty}\right)\right\|_{H^{l+1}}^{2}\right. \\
& \left.\quad+\left\|\frac{1}{\nu} \partial_{x_{1}}^{j}\left(\tilde{f}_{\infty}-\partial_{t} w_{\infty}+\frac{\tilde{\nu}}{\gamma} \nabla\left(\tilde{f}_{\infty}^{0}-\dot{\phi}_{\infty}\right)\right)\right\|_{H^{l}}^{2}\right\} \\
& \leq C\left\{\frac{1}{\nu^{2}}\left\|\partial_{t} \partial_{x_{1}}^{j} w_{\infty}\right\|_{H^{l}}^{2}+\frac{\nu^{2}+\tilde{\nu}^{2}}{\gamma^{2} \nu^{2}} \| \partial_{x_{1}}^{j} \dot{\phi}_{H^{l+1}}^{2}\right. \\
& \left.\quad+\frac{\nu^{2}+\tilde{\nu}^{2}}{\gamma^{2} \nu^{2}}\left\|\partial_{x_{1}}^{j} \tilde{f}_{\infty}^{0}\right\|_{H^{l+1}}^{2}+\frac{1}{\nu^{2}}\left\|\partial_{x_{1}}^{j} \tilde{f}_{\infty}\right\|_{H^{l}}^{2}\right\} .
\end{aligned}
$$

By $\frac{\nu^{2}}{\nu+\tilde{\nu}} \times(6.21)$ we obtain the desired result.

We are now in a position to prove (4.10).

Proof of (4.10). We compute $b_{1} \times\left\{(6.1)_{j=k=0}+(6.1)_{j=1, k=0}\right\}+(6.8)$ with a positive number $b_{1}$. Taking $b_{1}$ suitably large, we see that

$$
E_{0}(t)=b_{1}\left\|u_{\infty}(t)\right\|_{2}^{2}+D\left[w_{\infty}(t)\right]-2 \gamma\left(\phi_{\infty}(t), \operatorname{div} w_{\infty}(t)\right)
$$

is equivalent to

$$
\left\|u_{\infty}(t)\right\|_{2}^{2}+D\left[w_{\infty}(t)\right]
$$

and obtain

$$
\frac{1}{2} \frac{\mathrm{d}}{\mathrm{d} t} E_{1}(t)+\frac{1}{2} D_{1}(t) \leq C N_{1}(t)
$$

where

$$
\begin{aligned}
& E_{1}(t)=E_{0}(t)+b_{1}\left\|\partial_{x_{1}} u_{\infty}(t)\right\|_{2}^{2}, \\
& D_{1}(t)=b_{1} \sum_{j=0}^{1}\left(D\left[\partial_{x_{1}}^{j} w_{\infty}(t)\right]+\frac{\nu+\tilde{\nu}}{\gamma^{2}}\left\|\partial_{x_{1}}^{j} \dot{\phi}_{\infty}(t)\right\|_{2}^{2}\right)+\left\|\partial_{t} u_{\infty}(t)\right\|_{2}^{2}, \\
& N_{1}(t)=\sum_{j=0}^{1}\left|R_{j, 0}^{(1)}\right|+\left\|\tilde{f}_{\infty}^{0}\right\|_{2}^{2}+\left\|\tilde{f}_{\infty}\right\|_{2}^{2}+\left\|w \cdot \nabla \phi_{\infty}\right\|_{2}^{2} .
\end{aligned}
$$

We next consider $b_{2} \times(6.22)+(6.15)_{j=l=0}$. Then, with a suitably large $b_{2}>0$, we have

$$
\frac{1}{2} \frac{\mathrm{d}}{\mathrm{d} t} E_{2}(t)+\frac{1}{2} D_{2}(t) \leq C N_{2}(t)
$$


where

$$
\begin{aligned}
& E_{2}(t)=b_{2} E_{1}(t)+\left\|\partial_{x_{2}} \phi_{\infty}(t)\right\|_{2}^{2}, \\
& D_{2}(t)=\frac{b_{2}}{2} D_{1}(t)+\frac{\gamma^{2}}{\nu+\tilde{\nu}}\left\|\partial_{x_{2}} \phi_{\infty}(t)\right\|_{2}^{2}+\frac{\nu+\tilde{\nu}}{\gamma^{2}}\left\|\dot{\phi}_{\infty}(t)\right\|_{H^{1}}^{2}, \\
& N_{2}(t)=b_{2} N_{1}(t)+\left|R_{0,0}^{(2)}\right| .
\end{aligned}
$$

It then follows from $b_{3} \times(6.23)+(6.18)_{j=l=0}$ with a suitably large $b_{3}>0$ that

$$
\frac{1}{2} \frac{\mathrm{d}}{\mathrm{d} t} E_{3}(t)+\frac{1}{2} D_{3}(t) \leq C N_{3}(t)
$$

where

$$
\begin{aligned}
& E_{3}(t)=b_{3} E_{2}(t), \\
& D_{3}(t)=\frac{b_{3}}{2} D_{2}(t)+\frac{\gamma^{2}}{\nu+\tilde{\nu}}\left\|\partial_{x} \phi_{\infty}(t)\right\|_{2}^{2}+\frac{\nu^{2}}{\nu+\tilde{\nu}}\left\|\partial_{x}^{2} w_{\infty}(t)\right\|_{2}^{2}, \\
& N_{3}(t)=b_{3} N_{2}(t)+\frac{\nu+\tilde{\nu}}{\gamma^{2}}\left\|\tilde{f}_{\infty}^{0}\right\|_{H^{1}}^{2} .
\end{aligned}
$$

We next compute $(6.24)+b_{4} \times\left\{(6.1)_{j=2, k=0}+(6.1)_{j=0, k=1}\right\}+(6.15)_{j=1, l=0}$. Taking $b_{4}>0$ suitably large, we have

$$
\frac{1}{2} \frac{\mathrm{d}}{\mathrm{d} t} E_{4}(t)+\frac{1}{2} D_{4}(t) \leq C N_{4}(t)
$$

where

$$
\begin{aligned}
E_{4}(t)= & E_{3}(t)+b_{4}\left\{\left\|\partial_{x_{1}}^{2} u_{\infty}(t)\right\|_{2}^{2}+\left\|\partial_{t} u_{\infty}(t)\right\|_{2}^{2}\right\}+\left\|\partial_{x_{1}} \partial_{x_{2}} \phi_{\infty}(t)\right\|_{2}^{2}, \\
D_{4}(t)= & D_{3}(t)+b_{4}\left\{D\left[\partial_{x_{1}}^{2} w_{\infty}(t)\right]+D\left[\partial_{t} w_{\infty}(t)\right]\right\}+\frac{\gamma^{2}}{\nu+\tilde{\nu}}\left\|\partial_{x_{1}} \partial_{x_{2}} \phi_{\infty}(t)\right\|_{2}^{2} \\
& +\frac{\nu+\tilde{\nu}}{\gamma^{2}}\left\{\left\|\partial_{x_{1}} \dot{\phi}_{\infty}(t)\right\|_{H^{1}}^{2}+\left\|\partial_{t} \dot{\phi}_{\infty}(t)\right\|_{2}^{2}\right\}, \\
N_{4}(t)= & N_{3}(t)+\left|R_{2,0}^{(1)}(t)\right|+\left|R_{0,1}^{(1)}(t)\right|+\left|R_{1,0}^{(2)}(t)\right| .
\end{aligned}
$$

It then follows from $b_{5} \times(6.25)+(6.18)_{j=1, l=0}$ with a suitably large $b_{5}>0$ that

$$
\frac{1}{2} \frac{\mathrm{d}}{\mathrm{d} t} E_{5}(t)+\frac{1}{2} D_{5}(t) \leq C N_{5}(t)
$$

where

$$
\begin{aligned}
& E_{5}(t)=b_{5} E_{4}(t) \\
& D_{5}(t)=\frac{b_{5}}{2} D_{4}(t)+\frac{\gamma^{2}}{\nu+\tilde{\nu}}\left\|\partial_{x_{1}} \partial_{x} \phi_{\infty}(t)\right\|_{2}^{2}+\frac{\nu^{2}}{\nu+\tilde{\nu}}\left\|\partial_{x_{1}} \partial_{x}^{2} w_{\infty}(t)\right\|_{2}^{2}, \\
& N_{5}(t)=N_{4}(t)+\left\|\partial_{x_{1}} \tilde{f}_{\infty}^{0}(t)\right\|_{H^{1}}^{2}+\left\|\partial_{x_{1}} \tilde{f}_{\infty}(t)\right\|_{2}^{2} .
\end{aligned}
$$


We next consider $b_{6} \times(6.26)+(6.15)_{j=0, l=1}$. Then, with $b_{6}>0$ suitably large, we have

$$
\frac{1}{2} \frac{\mathrm{d}}{\mathrm{d} t} E_{6}(t)+\frac{1}{2} D_{6}(t) \leq C N_{6}(t)
$$

where

$$
\begin{aligned}
& E_{6}(t)=b_{6} E_{5}(t)+\left\|\partial_{x_{2}}^{2} \phi_{\infty}(t)\right\|_{2}^{2}, \\
& D_{6}(t)=\frac{b_{6}}{2} D_{5}(t)+\frac{\gamma^{2}}{\nu+\tilde{\nu}}\left\|\partial_{x_{2}}^{2} \phi_{\infty}(t)\right\|_{2}^{2}+\frac{\nu+\tilde{\nu}}{\gamma^{2}}\left\|\dot{\phi}_{\infty}(t)\right\|_{H^{2}}^{2}, \\
& N_{6}(t)=N_{5}(t)+\left|R_{0,1}^{(2)}(t)\right| .
\end{aligned}
$$

We then deduce from $b_{7} \times(6.27)+(6.18)_{j=0, l=1}$ with a suitably large $b_{7}>0$ that

$$
\frac{1}{2} \frac{\mathrm{d}}{\mathrm{d} t} E_{7}(t)+\frac{1}{2} D_{7}(t) \leq C N_{7}(t)
$$

where

$$
\begin{aligned}
& E_{7}(t)=b_{7} E_{6}(t), \\
& D_{7}(t)=\frac{b_{7}}{2} D_{6}(t)+\frac{\gamma^{2}}{\nu+\tilde{\nu}}\left\|\partial_{x}^{2} \phi_{\infty}(t)\right\|_{2}^{2}+\frac{\nu^{2}}{\nu+\tilde{\nu}}\left\|\partial_{x}^{3} w_{\infty}(t)\right\|_{2}^{2}, \\
& N_{7}(t)=N_{6}(t)+\left\|\tilde{f}_{\infty}^{0}(t)\right\|_{H^{2}}^{2}+\left\|\tilde{f}_{\infty}(t)\right\|_{H^{1}}^{2} .
\end{aligned}
$$

By (6.2) we have

$$
\left\|\partial_{t} \phi_{\infty}\right\|_{H^{1}}^{2} \leq C\left\{\gamma^{2}\left\|\nabla w_{\infty}\right\|_{H^{1}}^{2}+\gamma^{2}\left\|w \cdot \nabla \phi_{\infty}\right\|_{H^{1}}^{2}+\left\|\tilde{f}_{\infty}^{0}\right\|_{H^{1}}^{2}\right\} .
$$

We then deduce from $b_{8} \times(6.28)+(6.29)$ with a suitably large $b_{8}>0$ that

$$
\frac{1}{2} \frac{\mathrm{d}}{\mathrm{d} t} E_{8}(t)+\frac{1}{2} D_{8}(t) \leq C N_{8}(t)
$$

where

$$
\begin{aligned}
& E_{8}(t)=b_{8} E_{7}(t), \\
& D_{8}(t)=\frac{b_{8}}{2} D_{7}(t)+\left\|\partial_{t} \phi_{\infty}(t)\right\|_{H^{1}}^{2}, \\
& N_{8}(t)=N_{7}(t)+\left\|\left(w \cdot \nabla \phi_{\infty}\right)(t)\right\|_{H^{1}}^{2} .
\end{aligned}
$$

Note that $D_{8}(t)$ is equivalent to $D_{\infty}(t)$. By Remark 4.2, we have $D_{8}(t) \geq$ $c_{1} E_{8}(t)$ for some constant $c_{1}>0$, and hence,

$$
\frac{\mathrm{d}}{\mathrm{d} t} E_{8}(t)+c_{1} E_{8}(t)+D_{8}(t) \leq 2 C N_{8}(t) .
$$


This implies

$$
E_{8}(t)+\int_{0}^{t} e^{-c_{1}(t-\tau)} D_{8}(\tau) \mathrm{d} \tau \leq e^{-c_{1} t} E_{8}(0)+2 C \int_{0}^{t} e^{-c_{1}(t-\tau)} N_{8}(\tau) \mathrm{d} \tau .
$$

Since, by (6.3),

$$
\begin{aligned}
\nu \partial_{x_{2}}^{2} w_{\infty}^{1} & =\partial_{t} w_{\infty}^{1}-\nu \partial_{x_{1}}^{2} w_{\infty}^{1}-\tilde{\nu} \partial_{x_{1}}\left(\partial_{x_{1}} w_{\infty}^{1}+\partial_{x_{2}} w_{\infty}^{2}\right)+\gamma \partial_{x_{1}} \phi_{\infty}-f_{\infty}^{1} \\
(\nu+\tilde{\nu}) \partial_{x_{2}}^{2} w_{\infty}^{2} & =\partial_{t} w_{\infty}^{2}-\nu \partial_{x_{1}}^{2} w_{\infty}^{2}-\tilde{\nu} \partial_{x_{2}} \partial_{x_{1}} w_{\infty}^{1}+\gamma \partial_{x_{2}} \phi_{\infty}-f_{\infty}^{2}
\end{aligned}
$$

and

$$
\left\|\partial_{x_{1}} \partial_{x_{2}} w_{\infty}(t)\right\|_{2}^{2}=\left(\partial_{x_{1}}^{2} w_{\infty}(t), \partial_{x_{2}}^{2} w_{\infty}(t)\right) \leq\left\|\partial_{x_{1}}^{2} w_{\infty}(t)\right\|_{2}\left\|\partial_{x_{2}}^{2} w_{\infty}(t)\right\|_{2}
$$

we have

$$
\begin{aligned}
\left\|\partial_{x_{1}} \partial_{x_{2}} w_{\infty}(t)\right\|_{2}^{2}+\left\|\partial_{x_{2}}^{2} w(t)\right\|_{2}^{2} & \leq C\left\{E_{8}(t)+\left\|\tilde{f}_{\infty}(t)\right\|_{2}^{2}\right\} \\
& \leq C\left\{E_{8}(t)+(1+t)^{-\frac{5}{2}} M(t)^{4}\right\} .
\end{aligned}
$$

Therefore, we conclude that

$$
\begin{aligned}
& E_{\infty}(t)+\int_{0}^{t} e^{-a(t-\tau)} D_{\infty}(\tau) \mathrm{d} \tau \\
& \leq C\left\{e^{-a t} E_{\infty}(0)+(1+t)^{-\frac{5}{2}} M(t)^{4}+\int_{0}^{t} e^{-a(t-\tau)} N_{8}(\tau) \mathrm{d} \tau\right\}
\end{aligned}
$$

holds uniformly for $t \in[0, T]$ with $C>0$ independent of $T$. Here $a=a(\nu, \tilde{\nu}, \gamma)$ is a positive constant. We will see in section 7 that $N_{8}$ satisfies the estimate

$$
N_{8}(t) \leq C\left\{(1+t)^{-\frac{5}{2}} M(t)^{3}+(1+t)^{-\frac{1}{4}} M(t) D_{\infty}(t)\right\} .
$$

We thus obtain estimate (4.10) with $\mathcal{R}(t)=N_{8}(t)$ that satisfies (4.11). This completes the proof.

\section{Estimates on Nonlinearities}

In this section we estimate the nonlinearities to establish (4.11) for $\mathcal{R}(t)=$ $N_{8}(t)$.

By using the Gagliardo-Nirenberg-Sobolev inequality and Remark 4.2 we have the following estimates on $f^{0}=-\gamma \operatorname{div}(\phi w)$. 
Proposition 7.1 The following estimates hold uniformly for $t \in[0, T]$ with $C>0$ independent of $T$ :

$$
\begin{gathered}
\|\phi \operatorname{div} w\|_{H^{2}} \leq C\left\{(1+t)^{-\frac{5}{4}} M(t)^{2}+(1+t)^{-\frac{1}{2}} M(t) \sqrt{D_{\infty}(t)}\right\}, \\
\|w \cdot \nabla \phi\|_{H^{1}}+\left\|w \cdot \nabla \phi_{1}\right\|_{H^{2}} \leq C(1+t)^{-\frac{5}{4}} M(t)^{2}, \\
\left|\left(\operatorname{div} w,\left|\partial_{t}^{k} \partial_{x_{1}}^{j} \phi_{\infty}\right|^{2}\right)\right| \leq C\left\{(1+t)^{-\frac{5}{2}} M(t)^{3}+(1+t)^{-\frac{5}{2}} M(t) D_{\infty}(t)\right\}, \\
\left\|\left[\partial_{t}^{k} \partial_{x}^{j}, w\right] \cdot \nabla \phi_{\infty}\right\|_{2} \leq C\left\{(1+t)^{-2} M(t)^{2}+(1+t)^{-\frac{5}{4}} M(t) \sqrt{D_{\infty}(t)}\right\} \\
\text { for } 2 k+j \leq 2 \text { and } \\
\left\|\tilde{f}_{\infty}^{0}\right\|_{H^{2}} \leq C\left\{(1+t)^{-\frac{5}{4}} M(t)^{2}+(1+t)^{-\frac{1}{2}} M(t) \sqrt{D_{\infty}(t)}\right\}, \\
\left\|\partial_{t} \tilde{f}_{\infty}^{0}\right\|_{2} \leq C\left\{(1+t)^{-\frac{5}{4}} M(t)^{2}+(1+t)^{-\frac{1}{2}} M(t) \sqrt{D_{\infty}(t)}\right\} .
\end{gathered}
$$

We next consider the estimates for $\tilde{f}_{\infty}$. We recall that $\|\phi(t)\|_{\infty} \leq \frac{1}{2}$ whenever $\|u(t)\|_{H^{2}} \leq \varepsilon_{2}$, which follows from the Sobolev inequality $\|u(t)\|_{\infty} \leq$ $C\|u(t)\|_{H^{2}}$.

Proposition 7.2 If $\|u(t)\|_{H^{2}} \leq \varepsilon_{2}$ and $M(t) \leq 1$ for $t \in[0, T]$, then

$$
\begin{aligned}
& \left\|\tilde{f}_{\infty}\right\|_{2} \leq C(1+t)^{-\frac{5}{4}} M(t)^{2}, \\
& \left\|\tilde{f}_{\infty}\right\|_{H^{1}} \leq C\left\{(1+t)^{-\frac{5}{4}} M(t)^{2}+(1+t)^{-\frac{1}{2}} M(t) \sqrt{D_{\infty}(t)}\right\}, \\
& \left|\left(\partial_{t} \tilde{f}_{\infty}, \partial_{t} w_{\infty}\right)\right| \leq C\left\{(1+t)^{-\frac{5}{2}} M(t)^{3}+M(t) D_{\infty}(t)\right\}
\end{aligned}
$$

where $C>0$ is a constant independent of $T$.

Proof. The estimates (7.7)-(7.9) can be proved by using the GagliardoNirenberg-Sobolev inequality and Remark 4.2. We here estimate the term $\left(\partial_{t}\left(\frac{\phi}{1+\phi} \Delta w\right), \partial_{t} w_{\infty}\right)$ only, which appears in $(7.9)$. We set $g(\phi)=\frac{\phi}{1+\phi}$. Then

$$
\begin{aligned}
& \left|\left(\partial_{t}(g(\phi) \Delta w), \partial_{t} w_{\infty}\right)\right| \\
& =\left|-\left(\partial_{t}(\nabla(g(\phi)) \nabla w), \partial_{t} w_{\infty}\right)-\left(\partial_{t}(g(\phi) \nabla w), \partial_{t} \nabla w_{\infty}\right)\right| \\
& \leq\left|\left(\partial_{t}\left(g^{\prime}(\phi) \nabla \phi \nabla w\right), \partial_{t} w_{\infty}\right)\right|+\left|\left(\partial_{t}(g(\phi) \nabla w), \partial_{t} \nabla w_{\infty}\right)\right| \\
& =: \mathrm{I}+\mathrm{II} .
\end{aligned}
$$

The first term on the right is estimated as

$$
\begin{aligned}
\mathrm{I} \leq & \left|\left(g^{\prime \prime}(\phi) \partial_{t} \phi \nabla \phi \nabla w, \partial_{t} w_{\infty}\right)\right|+\left|\left(g^{\prime}(\phi) \partial_{t} \nabla \phi \nabla w, \partial_{t} w_{\infty}\right)\right| \\
& +\left|\left(g^{\prime}(\phi) \nabla \phi \partial_{t} \nabla w, \partial_{t} w_{\infty}\right)\right| \\
\leq & C\left\{\left\|\partial_{t} \phi\right\|_{4}\|\nabla \phi\|_{4}\|\nabla w\|_{\infty}\left\|\partial_{t} w_{\infty}\right\|_{2}+\left\|\partial_{t} \nabla \phi\right\|_{2}\|\nabla w\|_{\infty}\left\|\partial_{t} w_{\infty}\right\|_{2}\right. \\
& \left.+\|\nabla \phi\|_{4}\left\|\partial_{t} \nabla w\right\|_{2}\left\|\partial_{t} w_{\infty}\right\|_{4}\right\} \\
\leq & C\left\{\left\|\partial_{t} \phi\right\|_{H^{1}}\|\nabla \phi\|_{H^{1}}\|\nabla w\|_{H^{2}}\left\|\partial_{t} w_{\infty}\right\|_{2}+\left\|\partial_{t} \nabla \phi\right\|_{2}\|\nabla w\|_{H^{2}}\left\|\partial_{t} w_{\infty}\right\|_{2}\right. \\
& \left.+\|\nabla \phi\|_{H^{1}}\left\|\partial_{t} \nabla w\right\|_{2}\left\|\partial_{t} w_{\infty}\right\|_{H^{1}}\right\} \\
\leq & C\left\{(1+t)^{-\frac{5}{2}} M(t)^{3}+(1+t)^{-\frac{1}{2}} M(t) D_{\infty}(t)\right\} .
\end{aligned}
$$


As for II, we have

$$
\begin{aligned}
\mathrm{II} & \leq C\left\{\left\|g^{\prime}(\phi) \partial_{t} \phi \nabla w\right\|_{2}+\left\|g(\phi) \partial_{t} \nabla w\right\|_{2}\right\}\left\|\partial_{t} \nabla w_{\infty}\right\|_{2} \\
& \leq C\left\{\left\|\partial_{t} \phi\right\|_{4}\|\nabla w\|_{4}+\|g(\phi)\|_{\infty}\left\|\partial_{t} \nabla w\right\|_{2}\right\}\left\|\partial_{t} \nabla w_{\infty}\right\|_{2} \\
& \leq C\left\{\left\|\partial_{t} \phi\right\|_{H^{1}}\|\nabla w\|_{H^{1}}+\|\phi\|_{\infty}\left\|\partial_{t} \nabla w\right\|_{2}\right\}\left\|\partial_{t} \nabla w_{\infty}\right\|_{2} \\
& \leq C\left\{(1+t)^{-\frac{5}{2}} M(t)^{3}+M(t) D_{\infty}(t)\right\} .
\end{aligned}
$$

The other terms can be estimated similarly. This completes the proof.

The desired estimate for $\mathcal{R}(t)=N_{8}(t)$ follows from Propositions 7.1 and 7.2 .

\section{Asymptotic Behavior: Proof of Theorem 2.2}

In this section we prove Theorem 2.2. To this end we rewrite (1.1)-(1.2) in the form of conservation laws.

We set

$$
m=\rho v=\rho_{*}(1+\phi) v .
$$

Then (1.1)-(1.2) is written as

$$
\left\{\begin{array}{l}
\partial_{t} \rho+\operatorname{div} m=0, \\
\partial_{t} m-\mu \Delta\left(\frac{m}{\rho}\right)-\left(\mu+\mu^{\prime}\right) \nabla \operatorname{div}\left(\frac{m}{\rho}\right)+\nabla P(\rho)+\operatorname{div}\left(\frac{m \otimes m}{\rho}\right)=0,
\end{array}\right.
$$

and the boundary condition (1.3) is transformed into

$$
\left.\partial_{x_{2}}\left(\frac{m^{1}}{\rho}\right)\right|_{x_{2}=0,1}=0,\left.\quad m^{2}\right|_{x_{2}=0,1}=0 .
$$

We note that, from the proof of Theorem 2.1,

$$
\left\|m^{2}(t)\right\|_{2}=\left\|\gamma \rho(t) w^{2}(t)\right\|_{2}=O\left(t^{-\frac{5}{4}}\right) \text { as } t \rightarrow \infty .
$$

Therefore, to prove Theorem 2.2, it suffices to investigate the asymptotic behavior of ${ }^{\top}\left(\phi, m^{1}\right)$.

We decompose ${ }^{\top}\left(\phi, m^{1}\right)$ as

$$
\begin{gathered}
\phi=\Phi+\Phi_{\infty}, \quad \Phi=\phi_{1}=\tilde{P}_{1} \phi, \quad \Phi_{\infty}=\phi_{\infty}=\tilde{P}_{\infty} \phi \\
m^{1}=\rho_{*} \gamma\left(M+M_{\infty}\right), \quad M=\frac{1}{\rho_{*} \gamma} \tilde{P}_{1} m^{1}, \quad M_{\infty}=\frac{1}{\rho_{*} \gamma} \tilde{P}_{\infty} m^{1} .
\end{gathered}
$$


Note that $w^{1}=\frac{M+M_{\infty}}{1+\phi}$.

Applying $P_{1}$ to (8.1) and using (8.2), we have

$$
\left\{\begin{array}{l}
\partial_{t} \Phi+\gamma \partial_{x_{1}} M=0, \\
\partial_{t} M-(\nu+\tilde{\nu}) \partial_{x_{1}}^{2} M+\gamma \partial_{x_{1}} \Phi=\partial_{x_{1}} \tilde{P}_{1} g(U)+\partial_{x_{1}} \tilde{P}_{1} \tilde{g} .
\end{array}\right.
$$

Here $U=^{\top}(\Phi, M)$,

$$
\begin{aligned}
g(U)= & -\frac{\rho_{*} P^{\prime \prime}\left(\rho_{*}\right)}{2 \gamma} \Phi^{2}-\gamma M^{2}, \\
\tilde{g}=\tilde{g}(x, t)= & -(\nu+\tilde{\nu}) \partial_{x_{1}}\left(\phi w^{1}\right)-\frac{\rho_{*} P^{\prime \prime}\left(\rho_{*}\right)}{2 \gamma}\left(2 \Phi \Phi_{\infty}+\Phi_{\infty}^{2}\right) \\
& -\gamma\left(2 M M_{\infty}+M_{\infty}^{2}\right)+\gamma\left(\phi w^{1}\left(M+M_{\infty}\right)\right),
\end{aligned}
$$

where $\phi=\Phi+\Phi_{\infty}, w^{1}=\frac{M+M_{\infty}}{1+\phi}$.

We write (8.3) in the form

$$
\left\{\begin{array}{l}
\partial_{t} U+L_{0} U=\partial_{x_{1}} P_{0} G(U)+\partial_{x_{1}} P_{0} \tilde{G}, \quad U=P_{0} U, \\
\left.U\right|_{t=0}=P_{0} U_{0},
\end{array}\right.
$$

where $U_{0}={ }^{\top}\left(\phi_{0}, \frac{1}{\rho_{*} \gamma} m_{0}^{1}\right)={ }^{\top}\left(\phi_{0},\left(1+\phi_{0}\right) w_{0}^{1}\right)$,

$$
\begin{aligned}
L_{0} & =\mathcal{F}^{-1}\left(\begin{array}{cc}
0 & \gamma \partial_{x_{1}} \\
\gamma \partial_{x_{1}} & -(\nu+\tilde{\nu}) \partial_{x_{1}}^{2}
\end{array}\right), \\
G(U) & =\left(\begin{array}{c}
0 \\
g(U)
\end{array}\right), \quad \tilde{G}=\left(\begin{array}{l}
0 \\
\tilde{g}
\end{array}\right),
\end{aligned}
$$

and $P_{0}$ denotes the projection defined by

$$
P_{0}(U)=\left(\begin{array}{c}
\tilde{P}_{1} \Phi \\
\tilde{P}_{1} M
\end{array}\right)
$$

for $U=^{\top}(\Phi, M)$.

We see from Lemma 3.2 that

$$
e^{-t L_{0}}=\mathcal{F}^{-1}\left(e^{\lambda_{+} t} P_{+}+e^{\lambda_{-} t} P_{-}\right) \mathcal{F},
$$

where

$$
\begin{aligned}
& \lambda_{ \pm}=\lambda_{ \pm, 0}=-\frac{1}{2}(\nu+\tilde{\nu}) \xi^{2} \pm \frac{1}{2} \sqrt{(\nu+\tilde{\nu})^{2} \xi^{4}-4 \gamma^{2} \xi^{2}} \\
& P_{ \pm}= \pm \frac{1}{\lambda_{+}-\lambda_{-}}\left(\begin{array}{cc}
-\lambda_{\mp} & i \gamma \xi \\
i \gamma \xi & \lambda_{ \pm}
\end{array}\right) .
\end{aligned}
$$


We observe that, for $|\xi| \ll 1$,

$$
\begin{aligned}
& \lambda_{ \pm}=-\frac{\nu+\tilde{\nu}}{2} \xi^{2} \pm i \gamma \xi+O\left(\xi^{3}\right), \\
& P_{ \pm}=\frac{1}{2}\left(\begin{array}{cc}
1 & \pm 1 \\
\pm 1 & 1
\end{array}\right)(1+O(\xi)) .
\end{aligned}
$$

We define $S(t)$ and $S_{ \pm}(t)$ by

$$
\begin{aligned}
S(t) & =S_{+}(t)+S_{-}(t) \\
S_{ \pm}(t) & =\mathcal{F}^{-1} \hat{S}_{ \pm}(t) \mathcal{F} \\
\hat{S}_{ \pm}(t) & =\frac{1}{2} e^{-\frac{\nu+\tilde{\nu}}{2} \xi^{2} t \pm i \gamma \xi t}\left(\begin{array}{cc}
1 & \pm 1 \\
\pm 1 & 1
\end{array}\right) .
\end{aligned}
$$

Clearly, $e^{-t L_{0}} P_{0}$ has the same estimate as that for $e^{-t L} P_{1}$ such as (5.1). Furthermore, $e^{-t L_{0}} P_{0}$ is approximated by $S(t)$ in the following way. We define $\Pi_{0}$ by

$$
\Pi_{0} U_{0}=^{\top}\left(\left\langle\phi_{0}\right\rangle,\left\langle M_{0}\right\rangle\right) \text { for } U_{0}=^{\top}\left(\phi_{0}, M_{0}\right) .
$$

Note that $\Pi_{0} P_{0}=P_{0} \Pi_{0}=P_{0}$.

Lemma 8.1 The following estimates hold uniformly for $t>0$ :

(i) $\left\|\partial_{x_{1}}^{k} e^{-t L_{0}} P_{0} U_{0}\right\|_{2} \leq C(1+t)^{-\frac{1}{4}-\frac{k}{2}}\left\|U_{0}\right\|_{1}$,

(ii) $\left\|\partial_{x_{1}}^{k} S_{ \pm}(t) P_{0} U_{0}\right\|_{2} \leq C(1+t)^{-\frac{1}{4}-\frac{k}{2}}\left\|U_{0}\right\|_{1}$, $\left\|\partial_{x_{1}}^{k} S_{ \pm}(t) \Pi_{0}\left(I-P_{0}\right) U_{0}\right\|_{2} \leq C t^{-\frac{k}{2}} e^{-c_{0} t}\left\|U_{0}\right\|_{2}, \quad c_{0}=\frac{1}{2}(\nu+\tilde{\nu}) r_{0}^{2}$,

(iii) $\left\|\partial_{x_{1}}^{k}\left(e^{-t L_{0}}-S(t)\right) P_{0} U_{0}\right\|_{2} \leq C(1+t)^{-\frac{3}{4}-\frac{k}{2}}\left\|U_{0}\right\|_{1}$.

Proof. The estimates in (i), (ii) can be obtained by the same computation as in (5.1). As for (iii), since

$$
\begin{aligned}
& \left|e^{\lambda_{ \pm} t} P_{ \pm}-\hat{S}_{ \pm}(t)\right| \\
& =C\left\{\left|e^{-\frac{\nu+\tilde{\nu}}{2} \xi^{2} t \pm i \gamma \xi t}\left(e^{\lambda_{ \pm} t+\frac{\nu+\tilde{\nu}}{2} \xi^{2} t \mp i \gamma \xi t}-1\right)\right|+C|\xi| e^{R e \lambda_{ \pm} t}\right\} \\
& \leq C\left(|\xi|^{3} t+|\xi|\right) e^{-\frac{\nu+\tilde{\nu}}{4} \xi^{2} t}
\end{aligned}
$$

we have the desired estimate.

We denote by $U^{(0)}(t)={ }^{\top}\left(\phi^{(0)}\left(x_{1}, t\right), M^{(0), 1}\left(x_{1}, t\right)\right)$ the solution of the following integral equation:

$$
U^{(0)}(t)=S(t) \Pi_{0} U_{0}+\int_{0}^{t} S(t-\tau) \partial_{x_{1}} G\left(U^{(0)}(\tau)\right) \mathrm{d} \tau .
$$


We see from (8.4) that $U(t)$ is written as

$$
U(t)=e^{-t L_{0}} P_{0} U_{0}+\int_{0}^{t} e^{-(t-\tau) L_{0}} P_{0} \partial_{x_{1}}(G(U)+\tilde{G})(\tau) \mathrm{d} \tau .
$$

We will show that $U(t)$ is approximated by $U^{0}(t)$ as $t \rightarrow \infty$.

By Lemma 8.1, we have the following estimates for $U^{(0)}(t)$.

Proposition 8.2 If $\left\|U_{0}\right\|_{H^{2} \cap L^{1}} \ll 1$, then (8.5) has a unique solution $U^{(0)}(t)$ that satisfies

$$
\begin{gathered}
\left\|\partial_{x_{1}}^{k} U^{(0)}(t)\right\|_{2} \leq C(1+t)^{-\frac{1}{4}-\frac{k}{2}}\left\|U_{0}\right\|_{H^{2} \cap L^{1}}, \quad k=0,1,2 \\
\left\|\partial_{x_{1}}^{k} U^{(0)}(t)\right\|_{\infty} \leq C(1+t)^{-\frac{1}{2}-\frac{k}{2}}\left\|U_{0}\right\|_{H^{2} \cap L^{1}}, \quad k=0,1 .
\end{gathered}
$$

We have the following estimate for $U(t)-U^{(0)}(t)$.

Theorem 8.3 If $\left\|U_{0}\right\|_{H^{2} \cap L^{1}} \ll 1$, then

$$
\left\|\partial_{x_{1}}^{k}\left(U(t)-U^{(0)}(t)\right)\right\|_{2} \leq C(1+t)^{-\frac{3}{4}-\frac{k}{2}+\delta}\left\|U_{0}\right\|_{H^{2} \cap L^{1}}, \quad k=0,1,
$$

for any $\delta>0$.

Proof. We introduce $N(t)$ defined by

$$
N(t)=\sup _{0 \leq \tau \leq t}\left\{\sum_{k=0}^{1}(1+\tau)^{\frac{3}{4}+\frac{k}{2}-\delta}\left\|\partial_{x_{1}}^{k}\left(U(\tau)-U^{(0)}(\tau)\right)\right\|_{2}\right\} .
$$

It follows form (8.5)-(8.6) that $U(t)-U^{(0)}(t)$ is written as

$$
U(t)-U^{(0)}(t)=\sum_{j=0}^{4} I_{j}(t)
$$

where

$$
\begin{aligned}
& I_{0}(t)=\left(e^{-t L_{0}} P_{0}-S(t) \Pi_{0}\right) U_{0}, \\
& I_{1}(t)=\int_{0}^{t} S(t-\tau) P_{0} \partial_{x_{1}}\left(G(U(\tau))-G\left(U^{(0)}(\tau)\right)\right) \mathrm{d} \tau, \\
& I_{2}(t)=\int_{0}^{t}\left(e^{-(t-\tau) L_{0}}-S(t-\tau)\right) P_{0} \partial_{x_{1}} G(U(\tau)) \mathrm{d} \tau, \\
& I_{3}(t)=-\int_{0}^{t} S(t-\tau)\left(I-P_{0}\right) \partial_{x_{1}} G\left(U^{(0)}(\tau)\right) \mathrm{d} \tau, \\
& I_{4}(t)=\int_{0}^{t} e^{-(t-\tau) L_{0}} P_{0} \partial_{x_{1}} \tilde{G}(\tau) \mathrm{d} \tau .
\end{aligned}
$$


As for $I_{0}(t)$, we see from Lemma 8.1 (ii), (iii) that

$$
\begin{aligned}
\left\|\partial_{x_{1}}^{k} I_{0}(t)\right\|_{2} & \leq\left\|\partial_{x_{1}}^{k}\left(e^{-t L_{0}}-S(t)\right) P_{0} U_{0}\right\|_{2}+\left\|S(t) \Pi_{0}\left(I-P_{0}\right) \partial_{x_{1}}^{k} U_{0}\right\|_{2} \\
& \leq C(1+t)^{-\frac{3}{4}-\frac{k}{2}}\left\|U_{0}\right\|_{H^{1} \cap L^{1}}
\end{aligned}
$$

As for $I_{1}$, by Theorem 2.1 and Proposition 8.2, we have

$$
\begin{aligned}
\left\|G(U)-G\left(U^{(0)}\right)\right\|_{1} & \leq C\left\{\left\|\Phi^{2}-\left(\phi^{(0)}\right)^{2}\right\|_{1}+\left\|M^{2}-\left(M^{(0), 1}\right)^{2}\right\|_{1}\right\} \\
& \leq C\left\|U+U^{(0)}\right\|_{2}\left\|U-U^{(0)}\right\|_{2} \\
& \leq C(1+t)^{-1+\delta} N(t)\left\|U_{0}\right\|_{H^{2} \cap L^{1}},
\end{aligned}
$$

and similarly,

$$
\begin{aligned}
&\left\|\partial_{x_{1}}\left(G(U)-G\left(U^{(0)}\right)\right)\right\|_{1} \leq C\{\left\|\partial_{x_{1}}\left(U+U^{(0)}\right)\right\|_{2}\left\|U-U^{(0)}\right\|_{2} \\
&\left.+\left\|U+U^{(0)}\right\|_{2}\left\|\partial_{x_{1}}\left(U-U^{(0)}\right)\right\|_{2}\right\} \\
& \leq C(1+t)^{-\frac{3}{2}+\delta} N(t)\left\|U_{0}\right\|_{H^{2} \cap L^{1}}
\end{aligned}
$$

It then follows from Lemma 8.1 (ii) that

$$
\begin{aligned}
\left\|\partial_{x_{1}}^{k} I_{1}(t)\right\|_{2} \leq & C\left\{\int_{0}^{\frac{t}{2}}(1+t-\tau)^{-\frac{3}{4}-\frac{k}{2}}(1+\tau)^{-1+\delta} \mathrm{d} \tau\right. \\
& \left.+\int_{\frac{t}{2}}^{t}(1+t-\tau)^{-\frac{3}{4}}(1+\tau)^{-1-\frac{k}{2}+\delta} \mathrm{d} \tau\right\} N(t)\left\|U_{0}\right\|_{H^{2} \cap L^{1}} \\
\leq & C(1+t)^{-\frac{3}{4}-\frac{k}{2}+\delta} N(t)\left\|U_{0}\right\|_{H^{2} \cap L^{1}} .
\end{aligned}
$$

We next estimate $\partial_{x_{1}}^{k} I_{2}(t)$. Since

$$
\begin{aligned}
& \left\|\partial_{x_{1}} G(U)\right\|_{1} \leq C\|U\|_{2}\left\|\partial_{x_{1}} U\right\|_{2} \leq C(1+t)^{-1} M(t)^{2}, \\
& \left\|\partial_{x_{1}}^{2} G(U)\right\|_{1} \leq C\left\{\|U\|_{2}\left\|\partial_{x_{1}}^{2} U\right\|_{2}+\left\|\partial_{x_{1}} U\right\|_{2}^{2}\right\} \leq C(1+t)^{-\frac{3}{2}} M(t)^{2} .
\end{aligned}
$$

We see from Lemma 8.1 (iii) that

$$
\begin{aligned}
\left\|\partial_{x_{1}}^{k} I_{2}(t)\right\|_{2} \leq C\{ & \int_{0}^{\frac{t}{2}}(1+t-\tau)^{-\frac{3}{4}-\frac{k}{2}}(1+\tau)^{-1} \mathrm{~d} \tau \\
& \left.+\int_{\frac{t}{2}}^{t}(1+t-\tau)^{-\frac{3}{4}}(1+\tau)^{-1-\frac{k}{2}} \mathrm{~d} \tau\right\} M(t)^{2} \\
\leq C & (1+t)^{-\frac{3}{4}-\frac{k}{2}+\delta}\left\|U_{0}\right\|_{H^{2} \cap L^{1}}
\end{aligned}
$$


Concerning $I_{3}(t)$, we first observe that $\partial_{x_{1}} G\left(U^{(0)}\right)=\Pi_{0} \partial_{x_{1}} G\left(U^{(0)}\right)$ since $\partial_{x_{1}} G\left(U^{(0)}\right)$ depends only on $x_{1}$ and $t$. Furthermore, we have

$$
\begin{aligned}
\left\|\partial_{x_{1}} G\left(U^{(0)}\right)\right\|_{2} & \leq C\left\|U^{(0)}\right\|_{\infty}\left\|\partial_{x_{1}} U^{(0)}\right\|_{2} \leq C(1+t)^{-\frac{5}{4}}\left\|U_{0}\right\|_{H^{2} \cap L^{1}}^{2} \\
\left\|\partial_{x_{1}}^{2} G\left(U^{(0)}\right)\right\|_{2} & \leq C\left\{\left\|U^{(0)}\right\|_{\infty}\left\|\partial_{x_{1}}^{2} U^{(0)}\right\|_{2}+\left\|\partial_{x_{1}} U^{(0)}\right\|_{\infty}\left\|\partial_{x_{1}} U^{(0)}\right\|_{2}\right\} \\
& \leq C(1+t)^{-\frac{7}{4}}\left\|U_{0}\right\|_{H^{2} \cap L^{1}}^{2}
\end{aligned}
$$

It then follows from Lemma 8.1 (ii) that

$$
\begin{aligned}
\left\|\partial_{x_{1}}^{k} I_{3}(t)\right\|_{2} & \leq C \int_{0}^{t} e^{-c_{0}(t-\tau)}(1+\tau)^{-\frac{5}{4}-\frac{k}{2}} \mathrm{~d} \tau\left\|U_{0}\right\|_{H^{2} \cap L^{1}}^{2} \\
& \leq C(1+t)^{-\frac{5}{4}-\frac{k}{2}}\left\|U_{0}\right\|_{H^{2} \cap L^{1}}
\end{aligned}
$$

As for $I_{4}(t)$, we have

$$
\begin{aligned}
\|\tilde{G}\|_{1} & \leq C(1+t)^{-1} M(t)^{2}, \\
\left\|\partial_{x_{1}} \tilde{G}\right\|_{1} & \leq C(1+t)^{-\frac{3}{2}} M(t)^{2},
\end{aligned}
$$

and hence, similarly to the estimate for $\partial_{x_{1}}^{k} I_{2}(t)$,

$$
\left\|\partial_{x_{1}}^{k} I_{4}(t)\right\|_{2} \leq C(1+t)^{-\frac{3}{4}-\frac{k}{2}+\delta}\left\|U_{0}\right\|_{H^{2} \cap L^{1}} .
$$

This completes the proof.

Proof of Theorem 2.2. It suffices to show that $\| \partial_{x_{1}}^{k}\left(U^{(0)}-\chi_{+} \boldsymbol{b}_{+}-\right.$ $\left.\chi_{-} \boldsymbol{b}_{-}\right)(t) \|_{2}$ for $k=0,1$, where $\boldsymbol{b}_{ \pm}={ }^{\top}(1, \pm 1) \in \mathbb{R}^{2}$. Here $\chi_{ \pm}=\chi_{ \pm}\left(x_{1}, t\right)$ is the diffusion waves given in (1.10)-(1.12) with $c=\frac{1}{2}(a+b), a=-\frac{\rho_{*} P^{\prime \prime}\left(\rho_{*}\right)}{2 \gamma}, b=$ $-\gamma$. We follow the arguments in $[7,6]$. We write $U_{0}$ as

$$
U_{0}=U_{0+}+U_{0-}
$$

where

$$
U_{0 \pm}=\frac{1}{2}\left(\begin{array}{cc}
1 & \pm 1 \\
\pm 1 & 1
\end{array}\right) \Pi_{0} U_{0}=\frac{1}{2}\left\langle\phi_{0} \pm \frac{1}{\rho_{*} \gamma} m_{0}^{1}\right\rangle \boldsymbol{b}_{ \pm} .
$$

It then follows that

$$
U^{(0)}(t)=S_{+}(t) U_{0+}+S_{-}(t) U_{0-}+I_{1,+}(t)+I_{1,-}(t)
$$

where

$$
I_{1, \pm}(t)=\int_{0}^{t} S_{ \pm}(t-\tau) \partial_{x_{1}}\left(\begin{array}{c}
0 \\
a\left(\phi^{(0)}\right)^{2}+b\left(M^{(0), 1}\right)^{2}
\end{array}\right) \mathrm{d} t
$$


We write $I_{1, \pm}(t)$ as

$$
I_{1, \pm}= \pm \frac{1}{2} \int_{0}^{t} e^{-(t-\tau) L_{ \pm}} \partial_{x_{1}}\left(a\left(\phi^{(0)}\right)^{2}+b\left(M^{(0), 1}\right)^{2}\right) \mathrm{d} \tau \boldsymbol{b}_{ \pm},
$$

where

$$
e^{-t L_{ \pm}} u_{0}=\mathcal{F}^{-1}\left[e^{\left(-\frac{\nu+\tilde{\nu}}{2} \xi^{2} \pm i \gamma \xi\right) t} \hat{u}_{0}\right] .
$$

We note that $e^{-t L_{ \pm}}$satisfies the same estimates as those for $S_{ \pm}(t)$ in Lemma 8.1 (ii).

We define $V(t)={ }^{\top}(\eta(t), \zeta(t))$ by

$$
\begin{aligned}
U^{(0)}(t) & =\chi_{+}(t) \boldsymbol{b}_{+}+\chi_{-}(t) \boldsymbol{b}_{-}+V(t) \\
& =\left(\begin{array}{c}
\chi_{+}+\chi_{-}+\eta \\
\chi_{+}-\chi_{-}+\zeta
\end{array}\right),
\end{aligned}
$$

and introduce

$$
Y(t)=\sup _{0 \leq \tau \leq t}\left\{(1+\tau)^{\frac{1}{2}}\|V(\tau)\|_{2}+(1+\tau)\left\|\partial_{x_{1}} V(\tau)\right\|_{2}\right\} .
$$

We write

$$
\begin{aligned}
\left(\phi^{(0)}\right)^{2} & =\left(\chi_{+}+\chi_{-}+\eta\right)\left(\chi_{+}+\chi_{-}+\eta\right) \\
& =\chi_{+}^{2}+\chi_{-}^{2}+2 \chi_{+} \chi_{-}+\left(\chi_{+}+\chi_{+}\right) \eta+\eta\left(\chi_{+}+\chi_{-}+\eta\right) \\
& =\chi_{+}^{2}+\chi_{-}^{2}+2 \chi_{+} \chi_{-}+\left(\chi_{+}+\chi_{-}+\phi^{(0)}\right) \eta \\
& =\chi_{+}^{2}+\chi_{-}^{2}+2 \chi_{+} \chi_{-}+\sigma_{1} \eta
\end{aligned}
$$

and

$$
\begin{aligned}
\left(M^{(0), 1}\right)^{2} & =\chi_{+}^{2}+\chi_{-}^{2}-2 \chi_{+} \chi_{-}+\left(\chi_{+}-\chi_{-}+M^{(0), 1}\right) \zeta \\
& =\chi_{+}^{2}+\chi_{-}^{2}-2 \chi_{+} \chi_{-}+\sigma_{2} \zeta
\end{aligned}
$$

where $\sigma_{1}=\chi_{+}+\chi_{-}+\phi^{(0)}$ and $\sigma_{2}=\chi_{+}-\chi_{-}+M^{(0), 1}$. It then follows that $I_{1, \pm}(t)$ is written in the following forms

$$
\begin{aligned}
I_{1, \pm}(t)= \pm \frac{1}{2} \int_{0}^{t} e^{-(t-\tau) L_{ \pm}} & \partial_{x_{1}}\left((a+b)\left(\chi_{+}^{2}+\chi_{-}^{2}\right)+2(a-b) \chi_{+} \chi_{-}\right. \\
& \left.+a \sigma_{1} \eta+b \sigma_{2} \zeta\right) \mathrm{d} \tau \boldsymbol{b}_{ \pm} .
\end{aligned}
$$

Since $\chi_{ \pm}$satisfies

$$
\chi_{ \pm}(t)=e^{-t L_{ \pm}} \chi_{0 \pm} \pm \frac{a+b}{2} \int_{0}^{t} e^{-(t-\tau) L_{ \pm}} \partial_{x_{1}}\left(\chi_{ \pm}^{2}\right)(\tau) \mathrm{d} \tau
$$


where $\chi_{0 \pm}=\chi_{ \pm}(0)$, we see that

$$
\begin{aligned}
& V(t)=U^{(0)}(t)-\chi_{+}(t) \boldsymbol{b}_{+}-\chi_{-}(t) \boldsymbol{b}_{-} \\
& =S_{+}(t)\left(U_{0+}-\chi_{0+} \boldsymbol{b}_{+}\right)+S_{-}(t)\left(U_{0-}-\chi_{0-} \boldsymbol{b}_{-}\right)+I_{1,+}+I_{1,-} \\
& \quad-\frac{a+b}{2} \int_{0}^{t} e^{-(t-\tau) L_{+}} \partial_{x_{1}}\left(\chi_{+}^{2}\right)(\tau) \mathrm{d} \tau \boldsymbol{b}_{+}+\frac{a+b}{2} \int_{0}^{t} e^{-(t-\tau) L_{-}} \partial_{x_{1}}\left(\chi_{-}^{2}\right)(\tau) \mathrm{d} \tau \boldsymbol{b}_{-} \\
& =S_{+}(t)\left(U_{0+}-\chi_{0+} \boldsymbol{b}_{+}\right)+S_{-}(t)\left(U_{0-}-\chi_{0-} \boldsymbol{b}_{-}\right) \\
& \quad+\frac{1}{2}(a+b) \int_{0}^{t} e^{-(t-\tau) L_{+}} \partial_{x_{1}}\left(\chi_{-}^{2}\right)(\tau) \mathrm{d} \tau \boldsymbol{b}_{+} \\
& \quad-\frac{1}{2}(a+b) \int_{0}^{t} e^{-(t-\tau) L_{-}} \partial_{x_{1}}\left(\chi_{+}^{2}\right)(\tau) \mathrm{d} \tau \boldsymbol{b}_{-} \\
& \quad+(a-b) \int_{0}^{t} e^{-(t-\tau) L_{+}} \partial_{x_{1}}\left(\chi_{+} \chi_{-}\right)(\tau) \mathrm{d} \tau \boldsymbol{b}_{+} \\
& \quad-(a-b) \int_{0}^{t} e^{-(t-\tau) L_{-}} \partial_{x_{1}}\left(\chi_{+} \chi_{-}\right)(\tau) \mathrm{d} \tau \boldsymbol{b}_{-} \\
& \quad+\frac{1}{2} a \int_{0}^{t} e^{-(t-\tau) L_{+}} \partial_{x_{1}}\left(\sigma_{1} \eta\right)(\tau) \mathrm{d} \tau \mathrm{d} \tau \boldsymbol{b}_{+} \\
& \quad-\frac{1}{2} a \int_{0}^{t} e^{-(t-\tau) L_{-}} \partial_{x_{1}}\left(\sigma_{1} \eta\right)(\tau) \mathrm{d} \tau \boldsymbol{b}_{-} \\
& +\frac{1}{2} b \int_{0}^{t} e^{-(t-\tau) L_{+}} \partial_{x_{1}}\left(\sigma_{2} \zeta\right)(\tau) \mathrm{d} \tau \boldsymbol{b}_{+} \\
& \quad-\frac{1}{2} b \int_{0}^{t} e^{-(t-\tau) L_{-}} \partial_{x_{1}}\left(\sigma_{2} \zeta\right)(\tau) \mathrm{d} \tau \boldsymbol{b}_{-} .
\end{aligned}
$$


It then follows that

$$
\begin{aligned}
\left\|\partial_{x_{1}}^{k} V(t)\right\|_{L^{2}} \leq & \sum_{j= \pm}\left\|\partial_{x_{1}}^{k} S_{j}(t)\left(U_{0 j}-\chi_{0 j} \boldsymbol{b}_{j}\right)\right\|_{2} \\
& +C_{1}\left(\left\|\partial_{x_{1}}^{k} w_{+}(t)\right\|_{2}+\left\|\partial_{x_{1}}^{k} w_{-}(t)\right\|_{2}\right) \\
& +C_{2} \int_{0}^{t}\left\|\partial_{x_{1}}^{k} e^{-(t-\tau) L_{+}} \partial_{x_{1}}\left(\chi_{+} \chi_{-}\right)(\tau)\right\|_{2} \mathrm{~d} \tau \\
& +C_{3} \int_{0}^{t}\left\|\partial_{x_{1}}^{k} e^{-(t-\tau) L_{-}} \partial_{x_{1}}\left(\chi_{+} \chi_{-}\right)(\tau)\right\|_{2} \mathrm{~d} \tau \\
& +C_{4} \int_{0}^{t}\left\|\partial_{x_{1}}^{k} e^{-(t-\tau) L_{+}} \partial_{x_{1}}\left(\sigma_{1} \eta\right)(\tau)\right\|_{2} \mathrm{~d} \tau \\
& +C_{5} \int_{0}^{t}\left\|\partial_{x_{1}}^{k} e^{-(t-\tau) L_{-}} \partial_{x_{1}}\left(\sigma_{1} \eta\right)(\tau)\right\|_{2} \mathrm{~d} \tau \\
& +C_{6} \int_{0}^{t}\left\|\partial_{x_{1}}^{k} e^{-(t-\tau) L_{+}} \partial_{x_{1}}\left(\sigma_{2} \zeta\right)(\tau)\right\|_{2} \mathrm{~d} \tau \\
& +C_{7} \int_{0}^{t}\left\|\partial_{x_{1}}^{k} e^{-(t-\tau) L_{-}} \partial_{x_{1}}\left(\sigma_{2} \zeta\right)(\tau)\right\|_{2} \mathrm{~d} \tau \\
= & : \sum_{j= \pm}\left\|\partial_{x_{1}}^{k} S_{j}(t)\left(U_{0 j}-\chi_{0 j} \boldsymbol{b}_{j}\right)\right\|_{2}+\sum_{j=1}^{7} I_{j} .
\end{aligned}
$$

where

$$
\begin{gathered}
w_{ \pm}(t)=\int_{0}^{t} e^{-(t-\tau) L_{ \pm}} \partial_{x_{1}}\left(\chi_{\mp}^{2}\right)(\tau) \mathrm{d} \tau \\
C_{1}=\frac{1}{2}|a+b|, C_{2}=C_{3}=|a-b|, C_{4}=C_{5}=\frac{1}{2}|a|, C_{6}=C_{7}=\frac{1}{2}|b| .
\end{gathered}
$$

Since

$$
\begin{aligned}
& \int_{\mathbb{R}}\left(U_{0 \pm}-\chi_{0 \pm} \boldsymbol{b}_{ \pm}\right) \mathrm{d} x_{1} \\
& =\left[\frac{1}{2} \int_{\Omega}\left(\phi^{(0)} \pm \frac{1}{\rho_{*} \gamma} m_{0}^{1}\right) \mathrm{d} x-\int_{\mathbb{R}} \chi_{0 \pm} \mathrm{d} x_{1}\right] \boldsymbol{b}_{ \pm}=0
\end{aligned}
$$

we have

$$
\left\|\partial_{x_{1}}^{k} S_{ \pm}(t)\left(U_{0 \pm}-\chi_{0 \pm} \boldsymbol{b}_{ \pm}\right)\right\|_{2} \leq C t^{-\frac{1}{2}-\frac{k}{2}}\left\|u_{0}\right\|_{L_{1 / 2}^{1}} .
$$

As for $I_{1}$, we apply the estimates for $w_{ \pm}$by T.-P. Liu [8] (see also [6, Lemma 4.2]) to obtain

$$
I_{1} \leq C(1+t)^{-\frac{1}{2}-\frac{k}{2}}\left\|u_{0}\right\|_{H^{2} \cap L^{1}}^{2}
$$


We next estimate $I_{2}$. For $1 \leq p \leq \infty$ and $l \geq 0$, we have

$$
\left\|\partial_{x}^{l}\left(\chi_{+} \chi_{-}\right)(t)\right\|_{1} \leq C e^{-c t}\left\|u_{0}\right\|_{H^{2} \cap L^{1}}^{2} .
$$

See $[7,6]$ for estimate (8.9). It then follows from (8.9) and Lemma 8.1 (ii) that

$$
\begin{aligned}
I_{2} & \leq C \int_{0}^{t}(1+t-\tau)^{-\frac{3}{4}-\frac{k}{2}}\left(\left\|\chi_{+} \chi_{-}(\tau)\right\|_{1}+\left\|\partial_{x_{1}}^{k+1}\left(\chi_{+} \chi_{-}\right)(\tau)\right\|_{2}\right) \mathrm{d} \tau \\
& \leq C \int_{0}^{t}(1+t-\tau)^{-\frac{3}{4}-\frac{k}{2}} e^{-c \tau} \mathrm{d} \tau\left\|u_{0}\right\|_{H^{2} \cap L^{1}}^{2} \\
& \leq C(1+t)^{-\frac{3}{4}-\frac{k}{2}}\left\|u_{0}\right\|_{H^{2} \cap L^{1}}^{2} .
\end{aligned}
$$

Similarly, we have $I_{3} \leq C(1+t)^{-\frac{3}{4}-\frac{k}{2}}\left\|u_{0}\right\|_{H^{2} \cap L^{1}}^{2}$.

We next estimate $I_{4}$. By Lemma 8.1, we have

$$
\begin{aligned}
I_{4} \leq & C \int_{0}^{\frac{t}{2}}(1+t-\tau)^{-\frac{3}{4}-\frac{k}{2}}\left\|\sigma_{1} \eta(\tau)\right\|_{1} \mathrm{~d} \tau \\
& +C \int_{\frac{t}{2}}^{t}(1+t-\tau)^{-\frac{3}{4}}\left\|\partial_{x_{1}}^{k}\left(\sigma_{1} \eta\right)(\tau)\right\|_{2} \mathrm{~d} \tau \\
& +C \int_{0}^{t} e^{-c_{0}(t-\tau)}(t-\tau)^{-\frac{1}{2}}\left\|\partial_{x_{1}}^{k}\left(\sigma_{1} \eta\right)(\tau)\right\|_{2} \mathrm{~d} \tau \\
= & : I_{41}+I_{42}+I_{43} .
\end{aligned}
$$

By applying Proposition 8.2 and the following estimate

$$
\left\|\partial_{x_{1}}^{k} \chi_{ \pm}(t)\right\|_{2} \leq C(1+t)^{-\frac{1}{4}-\frac{k}{2}}\left\|u_{0}\right\|_{1}
$$

we see that $\left\|\sigma_{1}(\tau)\right\|_{2} \leq C(1+\tau)^{-\frac{1}{4}}\left\|u_{0}\right\|_{H^{2} \cap L^{1}}$. Since $\left\|\sigma_{1} \eta\right\|_{1} \leq\left\|\sigma_{1}\right\|_{2}\|\eta\|_{2}$, we have

$$
\begin{aligned}
I_{41} & =C Y(t) \int_{0}^{\frac{t}{2}}(1+t-\tau)^{-\frac{3}{4}-\frac{k}{2}}(1+\tau)^{-\frac{3}{4}} \mathrm{~d} \tau\left\|u_{0}\right\|_{H^{2} \cap L^{1}} \\
& \leq C(1+t)^{-\frac{1}{2}-\frac{k}{2}}\left\|u_{0}\right\|_{H^{2} \cap L^{1}} Y(t) .
\end{aligned}
$$

Similarly,

$$
\begin{aligned}
I_{42} & =C Y(t) \int_{\frac{t}{2}}^{t}(1+t-\tau)^{-\frac{3}{4}}(1+\tau)^{-\frac{3}{4}-\frac{k}{2}} \mathrm{~d} \tau\left\|u_{0}\right\|_{H^{2} \cap L^{1}} \\
& \leq C(1+t)^{-\frac{1}{2}-\frac{k}{2}}\left\|u_{0}\right\|_{H^{2} \cap L^{1}} Y(t),
\end{aligned}
$$


and

$$
\begin{aligned}
I_{43} & =C Y(t) \int_{0}^{t} e^{-c_{0}(t-\tau)}(t-\tau)^{-\frac{1}{2}}(1+\tau)^{-1-\frac{k}{2}} \mathrm{~d} \tau\left\|u_{0}\right\|_{H^{2} \cap L^{1}} \\
& \leq C(1+t)^{-\frac{1}{2}-\frac{k}{2}}\left\|u_{0}\right\|_{H^{2} \cap L^{1}} Y(t) .
\end{aligned}
$$

We thus obtain $I_{4} \leq C(1+t)^{-\frac{1}{2}-\frac{k}{2}}\left\|u_{0}\right\|_{H^{2} \cap L^{1}} Y(t)$. We can obtain the estimates for $I_{5}, I_{6}, I_{7}$ in a similar manner. It then follows that if $\left\|u_{0}\right\|_{H^{2} \cap L^{1}} \ll 1$, we have

$$
\left\|\partial_{x}^{k} V(t)\right\|_{2} \leq C(1+t)^{-\frac{1}{2}-\frac{k}{2}}\left\|u_{0}\right\|_{H^{2} \cap L^{1}}
$$

for $k=0,1$.

Since $w^{1}=\frac{1}{\rho_{* \gamma}} m^{1}-\phi w^{1}$, we have $w_{1}^{1}=M-\tilde{P}_{1}\left(\phi w^{1}\right)$, and so,

$$
u=\left(\begin{array}{c}
\phi_{1} \\
w_{1}^{1} \\
0
\end{array}\right)+\left(\begin{array}{c}
\phi_{\infty} \\
w_{\infty}^{1} \\
w^{2}
\end{array}\right)=\left(\begin{array}{c}
\Phi \\
M \\
0
\end{array}\right)+\left(\begin{array}{c}
\phi_{\infty} \\
-\tilde{P}_{1}\left(\phi w^{1}\right)+w_{\infty}^{1} \\
w^{2}
\end{array}\right)
$$

The desired estimate in Theorem 2.2 thus follows from Theorem 9.3 and (8.11). This completes the proof.

Acknowledgments. Y. Kagei was partly supported by JSPS KAKENHI Grant Number 24340028, 15K13449, 24224003, 16H03947.

\section{References}

[1] J. Brezina, Asymptotic behavior of solutions to the compressible NavierStokes equation around a time-periodic parallel flow, SIAM J. Math. Anal., 45 (2013), pp. 3514-3574.

[2] J. Brezina and Y. Kagei, Decay properties of solutions to the linearized compressible Navier-Stokes equation around time-periodic parallel flow, Math. Models Methods Appl. Sci., 22 (2012), 1250007, 53 pp.

[3] Y. Kagei, Large time behavior of solutions to the compressible NavierStokes equation in an infinite layer, Hiroshima Math. J., 38 (2008), pp. 95-124.

[4] Y. Kagei, Asymptotic behavior of solutions to the compressible NavierStokes equation around a parallel flow, Arch. Rational Mech. Anal. 205 (2012), pp. 585-650. 
[5] Y. Kagei and S. Kawashima, Local solvability of initial boundary value problem for a quasilinear hyperbolic-parabolic system, Journal of Hyperbolic Differential Equations, 3 (2006), pp.195-232.

[6] M. Kato, Y.-Z. Wang, S. Kawashima, Asymptotic behavior of solutions to the generalized cubic double dispersion equation in one space dimension, AIMS' Journals, 6 (2013), pp. 969-987.

[7] S. Kawashima, Large-time behaviour of solutions to hyperbolic-parabolic systems of conservation laws and applications, Proc. Roy. Soc. Edinburgh, 106A (1987), pp. 169-194.

[8] T.-P. Liu, "Hyperbolic and Viscous Conservation Laws," CBMS-NSF Regional Conference Sereies in Applied Math., vol. 72, SIAM, 2000.

[9] A. Matsumura, T. Nishida, Initial Boundary Value Problem for the Equations of Motion of Compressible Viscous and Heat-Conductive Fluids, Comm. Math. Phys. 89 (1983), pp. 445-464.

[10] T. Nishida, Equations of Motion of Compressible Viscous Fluids, Nonlinear Differential Equations, (1986), pp. 97-128. 


\section{List of MI Preprint Series, Kyushu University \\ The Global COE Program \\ Math-for-Industry Education \& Research Hub}

MI

MI2008-1 Takahiro ITO, Shuichi INOKUCHI \& Yoshihiro MIZOGUCHI

Abstract collision systems simulated by cellular automata

MI2008-2 Eiji ONODERA

The intial value problem for a third-order dispersive flow into compact almost Hermitian manifolds

MI2008-3 Hiroaki KIDO

On isosceles sets in the 4-dimensional Euclidean space

MI2008-4 Hirofumi NOTSU

Numerical computations of cavity flow problems by a pressure stabilized characteristiccurve finite element scheme

MI2008-5 Yoshiyasu OZEKI

Torsion points of abelian varieties with values in nfinite extensions over a p-adic field

MI2008-6 Yoshiyuki TOMIYAMA

Lifting Galois representations over arbitrary number fields

MI2008-7 Takehiro HIROTSU \& Setsuo TANIGUCHI

The random walk model revisited

MI2008-8 Silvia GANDY, Masaaki KANNO, Hirokazu ANAI \& Kazuhiro YOKOYAMA

Optimizing a particular real root of a polynomial by a special cylindrical algebraic decomposition

MI2008-9 Kazufumi KIMOTO, Sho MATSUMOTO \& Masato WAKAYAMA

Alpha-determinant cyclic modules and Jacobi polynomials

MI2008-10 Sangyeol LEE \& Hiroki MASUDA

Jarque-Bera Normality Test for the Driving Lévy Process of a Discretely Observed Univariate SDE

MI2008-11 Hiroyuki CHIHARA \& Eiji ONODERA

A third order dispersive flow for closed curves into almost Hermitian manifolds

MI2008-12 Takehiko KINOSHITA, Kouji HASHIMOTO and Mitsuhiro T. NAKAO

On the $L^{2}$ a priori error estimates to the finite element solution of elliptic problems with singular adjoint operator

MI2008-13 Jacques FARAUT and Masato WAKAYAMA

Hermitian symmetric spaces of tube type and multivariate Meixner-Pollaczek polynomials 
MI2008-14 Takashi NAKAMURA

Riemann zeta-values, Euler polynomials and the best constant of Sobolev inequality

MI2008-15 Takashi NAKAMURA

Some topics related to Hurwitz-Lerch zeta functions

MI2009-1 Yasuhide FUKUMOTO

Global time evolution of viscous vortex rings

MI2009-2 Hidetoshi MATSUI \& Sadanori KONISHI

Regularized functional regression modeling for functional response and predictors

MI2009-3 Hidetoshi MATSUI \& Sadanori KONISHI

Variable selection for functional regression model via the $L_{1}$ regularization

MI2009-4 Shuichi KAWANO \& Sadanori KONISHI

Nonlinear logistic discrimination via regularized Gaussian basis expansions

MI2009-5 Toshiro HIRANOUCHI \& Yuichiro TAGUCHII

Flat modules and Groebner bases over truncated discrete valuation rings

MI2009-6 Kenji KAJIWARA \& Yasuhiro OHTA

Bilinearization and Casorati determinant solutions to non-autonomous $1+1$ dimensional discrete soliton equations

MI2009-7 Yoshiyuki KAGEI

Asymptotic behavior of solutions of the compressible Navier-Stokes equation around the plane Couette flow

MI2009-8 Shohei TATEISHI, Hidetoshi MATSUI \& Sadanori KONISHI

Nonlinear regression modeling via the lasso-type regularization

MI2009-9 Takeshi TAKAISHI \& Masato KIMURA

Phase field model for mode III crack growth in two dimensional elasticity

MI2009-10 Shingo SAITO

Generalisation of Mack's formula for claims reserving with arbitrary exponents for the variance assumption

MI2009-11 Kenji KAJIWARA, Masanobu KANEKO, Atsushi NOBE \& Teruhisa TSUDA Ultradiscretization of a solvable two-dimensional chaotic map associated with the Hesse cubic curve

MI2009-12 Tetsu MASUDA

Hypergeometric $\tau$-functions of the q-Painlevé system of type $E_{8}^{(1)}$

MI2009-13 Hidenao IWANE, Hitoshi YANAMI, Hirokazu ANAI \& Kazuhiro YOKOYAMA A Practical Implementation of a Symbolic-Numeric Cylindrical Algebraic Decomposition for Quantifier Elimination

MI2009-14 Yasunori MAEKAWA

On Gaussian decay estimates of solutions to some linear elliptic equations and its applications 
MI2009-15 Yuya ISHIHARA \& Yoshiyuki KAGEI

Large time behavior of the semigroup on $L^{p}$ spaces associated with the linearized compressible Navier-Stokes equation in a cylindrical domain

MI2009-16 Chikashi ARITA, Atsuo KUNIBA, Kazumitsu SAKAI \& Tsuyoshi SAWABE

Spectrum in multi-species asymmetric simple exclusion process on a ring

MI2009-17 Masato WAKAYAMA \& Keitaro YAMAMOTO

Non-linear algebraic differential equations satisfied by certain family of elliptic functions

MI2009-18 Me Me NAING \& Yasuhide FUKUMOTO

Local Instability of an Elliptical Flow Subjected to a Coriolis Force

MI2009-19 Mitsunori KAYANO \& Sadanori KONISHI

Sparse functional principal component analysis via regularized basis expansions and its application

MI2009-20 Shuichi KAWANO \& Sadanori KONISHI

Semi-supervised logistic discrimination via regularized Gaussian basis expansions

MI2009-21 Hiroshi YOSHIDA, Yoshihiro MIWA \& Masanobu KANEKO

Elliptic curves and Fibonacci numbers arising from Lindenmayer system with symbolic computations

MI2009-22 Eiji ONODERA

A remark on the global existence of a third order dispersive flow into locally Hermitian symmetric spaces

MI2009-23 Stjepan LUGOMER \& Yasuhide FUKUMOTO

Generation of ribbons, helicoids and complex scherk surface in laser-matter Interactions

MI2009-24 Yu KAWAKAMI

Recent progress in value distribution of the hyperbolic Gauss map

MI2009-25 Takehiko KINOSHITA \& Mitsuhiro T. NAKAO

On very accurate enclosure of the optimal constant in the a priori error estimates for $H_{0}^{2}$-projection

MI2009-26 Manabu YOSHIDA

Ramification of local fields and Fontaine's property (Pm)

MI2009-27 Yu KAWAKAMI

Value distribution of the hyperbolic Gauss maps for flat fronts in hyperbolic threespace

MI2009-28 Masahisa TABATA

Numerical simulation of fluid movement in an hourglass by an energy-stable finite element scheme

MI2009-29 Yoshiyuki KAGEI \& Yasunori MAEKAWA

Asymptotic behaviors of solutions to evolution equations in the presence of translation and scaling invariance 
MI2009-30 Yoshiyuki KAGEI \& Yasunori MAEKAWA

On asymptotic behaviors of solutions to parabolic systems modelling chemotaxis

MI2009-31 Masato WAKAYAMA \& Yoshinori YAMASAKI

Hecke's zeros and higher depth determinants

MI2009-32 Olivier PIRONNEAU \& Masahisa TABATA

Stability and convergence of a Galerkin-characteristics finite element scheme of lumped mass type

MI2009-33 Chikashi ARITA

Queueing process with excluded-volume effect

MI2009-34 Kenji KAJIWARA, Nobutaka NAKAZONO \& Teruhisa TSUDA

Projective reduction of the discrete Painlevé system of type $\left(A_{2}+A_{1}\right)^{(1)}$

MI2009-35 Yosuke MIZUYAMA, Takamasa SHINDE, Masahisa TABATA \& Daisuke TAGAMI Finite element computation for scattering problems of micro-hologram using DtN map

MI2009-36 Reiichiro KAWAI \& Hiroki MASUDA

Exact simulation of finite variation tempered stable Ornstein-Uhlenbeck processes

MI2009-37 Hiroki MASUDA

On statistical aspects in calibrating a geometric skewed stable asset price model

MI2010-1 Hiroki MASUDA

Approximate self-weighted LAD estimation of discretely observed ergodic OrnsteinUhlenbeck processes

MI2010-2 Reiichiro KAWAI \& Hiroki MASUDA

Infinite variation tempered stable Ornstein-Uhlenbeck processes with discrete observations

MI2010-3 Kei HIROSE, Shuichi KAWANO, Daisuke MIIKE \& Sadanori KONISHI

Hyper-parameter selection in Bayesian structural equation models

MI2010-4 Nobuyuki IKEDA \& Setsuo TANIGUCHI

The Itô-Nisio theorem, quadratic Wiener functionals, and 1-solitons

MI2010-5 Shohei TATEISHI \& Sadanori KONISHI

Nonlinear regression modeling and detecting change point via the relevance vector machine

MI2010-6 Shuichi KAWANO, Toshihiro MISUMI \& Sadanori KONISHI

Semi-supervised logistic discrimination via graph-based regularization

MI2010-7 Teruhisa TSUDA

UC hierarchy and monodromy preserving deformation

MI2010-8 Takahiro ITO

Abstract collision systems on groups 
MI2010-9 Hiroshi YOSHIDA, Kinji KIMURA, Naoki YOSHIDA, Junko TANAKA \& Yoshihiro MIWA

An algebraic approach to underdetermined experiments

MI2010-10 Kei HIROSE \& Sadanori KONISHI

Variable selection via the grouped weighted lasso for factor analysis models

MI2010-11 Katsusuke NABESHIMA \& Hiroshi YOSHIDA

Derivation of specific conditions with Comprehensive Groebner Systems

MI2010-12 Yoshiyuki KAGEI, Yu NAGAFUCHI \& Takeshi SUDOU

Decay estimates on solutions of the linearized compressible Navier-Stokes equation around a Poiseuille type flow

MI2010-13 Reiichiro KAWAI \& Hiroki MASUDA

On simulation of tempered stable random variates

MI2010-14 Yoshiyasu OZEKI

Non-existence of certain Galois representations with a uniform tame inertia weight

MI2010-15 Me Me NAING \& Yasuhide FUKUMOTO

Local Instability of a Rotating Flow Driven by Precession of Arbitrary Frequency

MI2010-16 Yu KAWAKAMI \& Daisuke NAKAJO

The value distribution of the Gauss map of improper affine spheres

MI2010-17 Kazunori YASUTAKE

On the classification of rank 2 almost Fano bundles on projective space

MI2010-18 Toshimitsu TAKAESU

Scaling limits for the system of semi-relativistic particles coupled to a scalar bose field

MI2010-19 Reiichiro KAWAI \& Hiroki MASUDA

Local asymptotic normality for normal inverse Gaussian Lévy processes with highfrequency sampling

MI2010-20 Yasuhide FUKUMOTO, Makoto HIROTA \& Youichi MIE

Lagrangian approach to weakly nonlinear stability of an elliptical flow

MI2010-21 Hiroki MASUDA

Approximate quadratic estimating function for discretely observed Lévy driven SDEs with application to a noise normality test

MI2010-22 Toshimitsu TAKAESU

A Generalized Scaling Limit and its Application to the Semi-Relativistic Particles System Coupled to a Bose Field with Removing Ultraviolet Cutoffs

MI2010-23 Takahiro ITO, Mitsuhiko FUJIO, Shuichi INOKUCHI \& Yoshihiro MIZOGUCHI Composition, union and division of cellular automata on groups

MI2010-24 Toshimitsu TAKAESU

A Hardy's Uncertainty Principle Lemma in Weak Commutation Relations of HeisenbergLie Algebra 
MI2010-25 Toshimitsu TAKAESU

On the Essential Self-Adjointness of Anti-Commutative Operators

MI2010-26 Reiichiro KAWAI \& Hiroki MASUDA

On the local asymptotic behavior of the likelihood function for Meixner Lévy processes under high-frequency sampling

MI2010-27 Chikashi ARITA \& Daichi YANAGISAWA

Exclusive Queueing Process with Discrete Time

MI2010-28 Jun-ichi INOGUCHI, Kenji KAJIWARA, Nozomu MATSUURA \& Yasuhiro OHTA Motion and Bäcklund transformations of discrete plane curves

MI2010-29 Takanori YASUDA, Masaya YASUDA, Takeshi SHIMOYAMA \& Jun KOGURE On the Number of the Pairing-friendly Curves

MI2010-30 Chikashi ARITA \& Kohei MOTEGI

Spin-spin correlation functions of the $q$-VBS state of an integer spin model

MI2010-31 Shohei TATEISHI \& Sadanori KONISHI

Nonlinear regression modeling and spike detection via Gaussian basis expansions

MI2010-32 Nobutaka NAKAZONO

Hypergeometric $\tau$ functions of the $q$-Painlevé systems of type $\left(A_{2}+A_{1}\right)^{(1)}$

MI2010-33 Yoshiyuki KAGEI

Global existence of solutions to the compressible Navier-Stokes equation around parallel flows

MI2010-34 Nobushige KUROKAWA, Masato WAKAYAMA \& Yoshinori YAMASAKI

Milnor-Selberg zeta functions and zeta regularizations

MI2010-35 Kissani PERERA \& Yoshihiro MIZOGUCHI

Laplacian energy of directed graphs and minimizing maximum outdegree algorithms

MI2010-36 Takanori YASUDA

CAP representations of inner forms of $S p(4)$ with respect to Klingen parabolic subgroup

MI2010-37 Chikashi ARITA \& Andreas SCHADSCHNEIDER

Dynamical analysis of the exclusive queueing process

MI2011-1 Yasuhide FUKUMOTO\& Alexander B. SAMOKHIN

Singular electromagnetic modes in an anisotropic medium

MI2011-2 Hiroki KONDO, Shingo SAITO \& Setsuo TANIGUCHI

Asymptotic tail dependence of the normal copula

MI2011-3 Takehiro HIROTSU, Hiroki KONDO, Shingo SAITO, Takuya SATO, Tatsushi TANAKA \& Setsuo TANIGUCHI

Anderson-Darling test and the Malliavin calculus

MI2011-4 Hiroshi INOUE, Shohei TATEISHI \& Sadanori KONISHI

Nonlinear regression modeling via Compressed Sensing 
MI2011-5 Hiroshi INOUE

Implications in Compressed Sensing and the Restricted Isometry Property

MI2011-6 Daeju KIM \& Sadanori KONISHI

Predictive information criterion for nonlinear regression model based on basis expansion methods

MI2011-7 Shohei TATEISHI, Chiaki KINJYO \& Sadanori KONISHI

Group variable selection via relevance vector machine

MI2011-8 Jan BREZINA \& Yoshiyuki KAGEI

Decay properties of solutions to the linearized compressible Navier-Stokes equation around time-periodic parallel flow

Group variable selection via relevance vector machine

MI2011-9 Chikashi ARITA, Arvind AYYER, Kirone MALLICK \& Sylvain PROLHAC

Recursive structures in the multispecies TASEP

MI2011-10 Kazunori YASUTAKE

On projective space bundle with nef normalized tautological line bundle

MI2011-11 Hisashi ANDO, Mike HAY, Kenji KAJIWARA \& Tetsu MASUDA

An explicit formula for the discrete power function associated with circle patterns of Schramm type

MI2011-12 Yoshiyuki KAGEI

Asymptotic behavior of solutions to the compressible Navier-Stokes equation around a parallel flow

MI2011-13 Vladimír CHALUPECKÝ \& Adrian MUNTEAN

Semi-discrete finite difference multiscale scheme for a concrete corrosion model: approximation estimates and convergence

MI2011-14 Jun-ichi INOGUCHI, Kenji KAJIWARA, Nozomu MATSUURA \& Yasuhiro OHTA Explicit solutions to the semi-discrete modified KdV equation and motion of discrete plane curves

MI2011-15 Hiroshi INOUE

A generalization of restricted isometry property and applications to compressed sensing

MI2011-16 Yu KAWAKAMI

A ramification theorem for the ratio of canonical forms of flat surfaces in hyperbolic three-space

MI2011-17 Naoyuki KAMIYAMA

Matroid intersection with priority constraints

MI2012-1 Kazufumi KIMOTO \& Masato WAKAYAMA

Spectrum of non-commutative harmonic oscillators and residual modular forms

MI2012-2 Hiroki MASUDA

Mighty convergence of the Gaussian quasi-likelihood random fields for ergodic Levy driven SDE observed at high frequency 
MI2012-3 Hiroshi INOUE

A Weak RIP of theory of compressed sensing and LASSO

MI2012-4 Yasuhide FUKUMOTO \& Youich MIE

Hamiltonian bifurcation theory for a rotating flow subject to elliptic straining field

MI2012-5 Yu KAWAKAMI

On the maximal number of exceptional values of Gauss maps for various classes of surfaces

MI2012-6 Marcio GAMEIRO, Yasuaki HIRAOKA, Shunsuke IZUMI, Miroslav KRAMAR, Konstantin MISCHAIKOW \& Vidit NANDA

Topological Measurement of Protein Compressibility via Persistence Diagrams

MI2012-7 Nobutaka NAKAZONO \& Seiji NISHIOKA

Solutions to a $q$-analog of Painlevé III equation of type $D_{7}^{(1)}$

MI2012-8 Naoyuki KAMIYAMA

A new approach to the Pareto stable matching problem

MI2012-9 Jan BREZINA \& Yoshiyuki KAGEI

Spectral properties of the linearized compressible Navier-Stokes equation around time-periodic parallel flow

MI2012-10 Jan BREZINA

Asymptotic behavior of solutions to the compressible Navier-Stokes equation around a time-periodic parallel flow

MI2012-11 Daeju KIM, Shuichi KAWANO \& Yoshiyuki NINOMIYA

Adaptive basis expansion via the extended fused lasso

MI2012-12 Masato WAKAYAMA

On simplicity of the lowest eigenvalue of non-commutative harmonic oscillators

MI2012-13 Masatoshi OKITA

On the convergence rates for the compressible

Navier- Stokes equations with potential force

MI2013-1 Abuduwaili PAERHATI \& Yasuhide FUKUMOTO

A Counter-example to Thomson-Tait-Chetayev's Theorem

MI2013-2 Yasuhide FUKUMOTO \& Hirofumi SAKUMA

A unified view of topological invariants of barotropic and baroclinic fluids and their application to formal stability analysis of three-dimensional ideal gas flows

MI2013-3 Hiroki MASUDA

Asymptotics for functionals of self-normalized residuals of discretely observed stochastic processes

MI2013-4 Naoyuki KAMIYAMA

On Counting Output Patterns of Logic Circuits

MI2013-5 Hiroshi INOUE

RIPless Theory for Compressed Sensing 
MI2013-6 Hiroshi INOUE

Improved bounds on Restricted isometry for compressed sensing

MI2013-7 Hidetoshi MATSUI

Variable and boundary selection for functional data via multiclass logistic regression modeling

MI2013-8 Hidetoshi MATSUI

Variable selection for varying coefficient models with the sparse regularization

MI2013-9 Naoyuki KAMIYAMA

Packing Arborescences in Acyclic Temporal Networks

MI2013-10 Masato WAKAYAMA

Equivalence between the eigenvalue problem of non-commutative harmonic oscillators and existence of holomorphic solutions of Heun's differential equations, eigenstates degeneration, and Rabi's model

MI2013-11 Masatoshi OKITA

Optimal decay rate for strong solutions in critical spaces to the compressible Navier-

Stokes equations

MI2013-12 Shuichi KAWANO, Ibuki HOSHINA, Kazuki MATSUDA \& Sadanori KONISHI

Predictive model selection criteria for Bayesian lasso

MI2013-13 Hayato CHIBA

The First Painleve Equation on the Weighted Projective Space

MI2013-14 Hidetoshi MATSUI

Variable selection for functional linear models with functional predictors and a functional response

MI2013-15 Naoyuki KAMIYAMA

The Fault-Tolerant Facility Location Problem with Submodular Penalties

MI2013-16 Hidetoshi MATSUI

Selection of classification boundaries using the logistic regression

MI2014-1 Naoyuki KAMIYAMA

Popular Matchings under Matroid Constraints

MI2014-2 Yasuhide FUKUMOTO \& Youichi MIE

Lagrangian approach to weakly nonlinear interaction of Kelvin waves and a symmetrybreaking bifurcation of a rotating flow

MI2014-3 Reika AOYAMA

Decay estimates on solutions of the linearized compressible Navier-Stokes equation around a Parallel flow in a cylindrical domain

MI2014-4 Naoyuki KAMIYAMA

The Popular Condensation Problem under Matroid Constraints 
MI2014-5 Yoshiyuki KAGEI \& Kazuyuki TSUDA

Existence and stability of time periodic solution to the compressible Navier-Stokes equation for time periodic external force with symmetry

MI2014-6 This paper was withdrawn by the authors.

MI2014-7 Masatoshi OKITA

On decay estimate of strong solutions in critical spaces for the compressible NavierStokes equations

MI2014-8 Rong ZOU \& Yasuhide FUKUMOTO

Local stability analysis of azimuthal magnetorotational instability of ideal MHD flows

MI2014-9 Yoshiyuki KAGEI \& Naoki MAKIO

Spectral properties of the linearized semigroup of the compressible Navier-Stokes equation on a periodic layer

MI2014-10 Kazuyuki TSUDA

On the existence and stability of time periodic solution to the compressible NavierStokes equation on the whole space

MI2014-11 Yoshiyuki KAGEI \& Takaaki NISHIDA

Instability of plane Poiseuille flow in viscous compressible gas

MI2014-12 Chien-Chung HUANG, Naonori KAKIMURA \& Naoyuki KAMIYAMA

Exact and approximation algorithms for weighted matroid intersection

MI2014-13 Yusuke SHIMIZU

Moment convergence of regularized least-squares estimator for linear regression model

MI2015-1 Hidetoshi MATSUI \& Yuta UMEZU

Sparse regularization for multivariate linear models for functional data

MI2015-2 Reika AOYAMA \& Yoshiyuki KAGEI

Spectral properties of the semigroup for the linearized compressible Navier-Stokes equation around a parallel flow in a cylindrical domain

MI2015-3 Naoyuki KAMIYAMA

Stable Matchings with Ties, Master Preference Lists, and Matroid Constraints

MI2015-4 Reika AOYAMA \& Yoshiyuki KAGEI

Large time behavior of solutions to the compressible Navier-Stokes equations around a parallel flow in a cylindrical domain

MI2015-5 Kazuyuki TSUDA

Existence and stability of time periodic solution to the compressible Navier-StokesKorteweg system on $R^{3}$

MI2015-6 Naoyuki KAMIYAMA

Popular Matchings with Ties and Matroid Constraints 
MI2015-7 Shoichi EGUCHI \& Hiroki MASUDA

Quasi-Bayesian model comparison for LAQ models

MI2015-8 Yoshiyuki KAGEI \& Ryouta OOMACHI

Stability of time periodic solution of the Navier-Stokes equation on the half-space under oscillatory moving boundary condition

MI2016-1 Momonari KUDO

Analysis of an algorithm to compute the cohomology groups of coherent sheaves and its applications

MI2016-2 Yoshiyuki KAGEI \& Masatoshi OKITA

Asymptotic profiles for the compressible Navier-Stokes equations on the whole space

MI2016-3 Shota ENOMOTO \& Yoshiyuki KAGEI

Asymptotic behavior of the linearized semigroup at space-periodic stationary solution of the compressible Navier-Stokes equation

MI2016-4 Hiroki MASUDA

Non-Gaussian quasi-likelihood estimation of locally stable SDE

MI2016-5 Yoshiyuki KAGEI \& Takaaki NISHIDA

On Chorin's method for stationary solutions of the Oberbeck-Boussinesq equation

MI2016-6 Hayato WAKI \& Florin NAE

Boundary modeling in model-based calibration for automotive engines via the vertex representation of the convex hulls

MI2016-7 Kazuyuki TSUDA

Time periodic problem for the compressible Navier-Stokes equation on $R^{2}$ with antisymmetry

MI2016-8 Abulizi AIHAITI, Shota ENOMOTO \& Yoshiyuki KAGEI

Large time behavior of solutions to the compressible Navier-Stokes equations in an infinite layer under slip boundary condition 